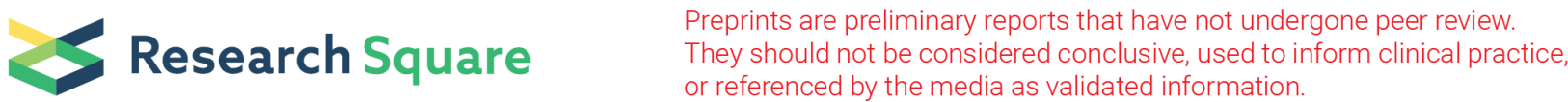

\section{Effect of Maternal Positioning During Cardiopulmonary Resuscitation: A Systematic Review and Meta-analyses}

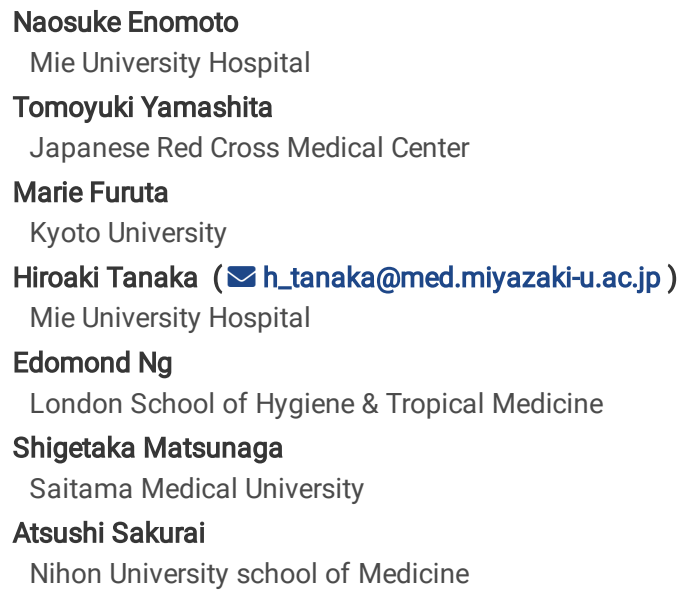

Version of Record: A version of this preprint was published at BMC Pregnancy and Childbirth on February 25th, 2022. See the published version at https://doi.org/10.1186/s12884-021-04334-y. 


\section{Abstract}

Background: Although rare, cardiac arrest during pregnancy is the leading cause of maternal death. Recently, its incidence has been increasing worldwide because more pregnant women have risk factors. The provision of early, high-quality cardiopulmonary resuscitation (CPR) plays a major role in the increased likelihood of survival; therefore, clinicians should be familiar with its management. Due to the aortocaval compression caused by the gravid uterus, clinical guidelines often emphasise the importance of maternal positioning during CPR, but there has been little evidence regarding which position is most effective.

Methods: We searched the Cochrane Central Register of Controlled Trials, MEDLINE, Embase, and OpenGrey (updated on April 3, 2021). We included clinical trials and observational studies with reported outcomes related to successful resuscitations.

Results: We included eight studies out of the 1,490 screened. All of these were simulation-based cross-over randomised controlled trials examining the quality of chest compressions. No data were available about the survival rates of mothers or fetuses/neonates. Meta-analyses indicated that resuscitation of pregnant women in the $27^{\circ}-30^{\circ}$ left-lateral tilt position lowered the quality of chest compressions, and the rates of correct compression depth and correct hand position decreased by $17 \%$ and $9 \%$, respectively when compared with resuscitation in the supine position. Inexperienced clinicians faced difficulties in performing chest compressions in the left-lateral tilt position.

Conclusions: Given that manual left uterine displacement allows the patient to remain supine, the resuscitation of women in the supine position using manual left uterine displacement should continue to be supported. Further research is needed to fill knowledge gaps regarding the effects of maternal positioning on clinical outcomes, such as survival rates following maternal cardiac arrest.

\section{Introduction}

Cardiac arrest during pregnancy is rare but life-threatening and involves the lives of two patients: the mother and the fetus [1]. It remains one of the leading causes of maternal deaths worldwide [2, 3]. Nationwide population-based studies from the United States, Canada, the United Kingdom and the Netherlands indicate the incidence of maternal cardiac arrest during pregnancy is approximately 1 in 12,000 to 1 in 36,000 [4-7]. The incidence of maternal cardiac arrest and related maternal mortality have increased in several countries over the past 30 years $[5,8,9]$. This increase could be explained partially by more women with risk factors (rising maternal age, obesity and preexisting chronic medical conditions) becoming pregnant [3, 9]. Common causes of maternal cardiac arrest and mortality include anaesthesia complications, bleeding, cardiovascular disease, embolism, uterine atony and hypertension/preeclampsia/eclampsia $[8,10,11]$. Studies indicate some clinical incidents of cardiac arrest among pregnant women, who are typically younger than other patients who experience cardiac arrest, are more responsive to prompt and appropriate medical treatment than most patients requiring cardiopulmonary resuscitation (CPR) [12].

The rate of maternal survival to hospital discharge for in-hospital maternal cardiac arrest is estimated to be as high as 59\% [5, 6], whereas the corresponding figure for maternal cardiac arrest occurring in out-of-hospital settings is much lower, at around 17\% [13]. The provision of early, high-quality CPR plays a significant role in increasing the likelihood of survival [14]. Although the resuscitation of a pregnant woman is similar to the standard resuscitation of adults, the physiological changes that occur during pregnancy impose additional clinical challenges $[8,14,15]$. Aortocaval compression occurs beginning around 20 weeks of gestation, when the growth of the uteroplacental unit compresses the aorta, inferior vena cava or both in the supine position [16]. Such compression can reduce cardiac output by as much as $30-40 \%$ [17]. During CPR, manual chest compressions could produce approximately $30 \%$ of the normal cardiac output for the nonpregnant situation [18]. Aortocaval compression in late pregnancy further reduces cardiac output to around $10 \%$ of the nonpregnant cardiac output $[19,20]$.

Clinical guidelines [21-26] recommend relief of aortocaval compression during maternal resuscitation. However, there is no consensus on the best strategy to relieve aortocaval compression during maternal resuscitation. Thus, the latest Royal College of Obstetricians and Gynaecologists guidelines on 'maternal collapse in pregnancy and the puerperium' recommended future researchers investigate the effectiveness of CPR with manual uterine displacement versus maternal tilt [19], both of which are considered beneficial in relieving aortocaval compression during chest compressions. A Cochrane systematic review on maternal position during caesarean section for preventing maternal and neonatal complications has been published [16], but the result was based on nonarrest pregnant women, and in light of the quality of chest compression, some strategies that could be effective in relieving aortocaval compression for nonarrest pregnant patients might not be the best option for pregnant women in cardiac arrest.

No current or planned systematic reviews regarding the effects of maternal positioning or strategies were identified in a search of the Cochrane Library, International Prospective Register of Systematic Reviews (PROSPERO) or the Joanna Briggs Institute. Therefore, our systematic review aimed to synthesise the evidence to evaluate the effect of maternal positioning and other strategies during resuscitation to determine which is most effective in improving outcomes following maternal cardiac arrest. Our findings will contribute to evidence-based decision-making for clinicians and provide a basis for the formation of national and international guidelines on the resuscitation of pregnant women.

\section{Materials And Methods}

Our review was registered in PROSPERO (CRD42020208177) and conducted in accordance with Preferred Reporting Items for Systematic Reviews and MetaAnalyses (PRISMA) guidelines [27].

\section{Search Strategy}

We searched the Cochrane Central Register of Controlled Trials (CENTRAL), MEDLINE, Embase and OpenGrey databases for relevant studies on 16 November 2019, and we updated them on 3 April 2021. We did not restrict the publication year. We also checked the reference lists of all included studies and relevant 
existing systematic reviews for additional studies. We used subject headings in combination with key words. We devised three sets of search terms: (i) population of interest (pregnant women), (ii) health condition of interest (cardiac arrest) and (iii) intervention (or exposure) evaluated (Table 1).

Table 1

Search strategy (Medline OvidSP) 1946 to April 2021. 


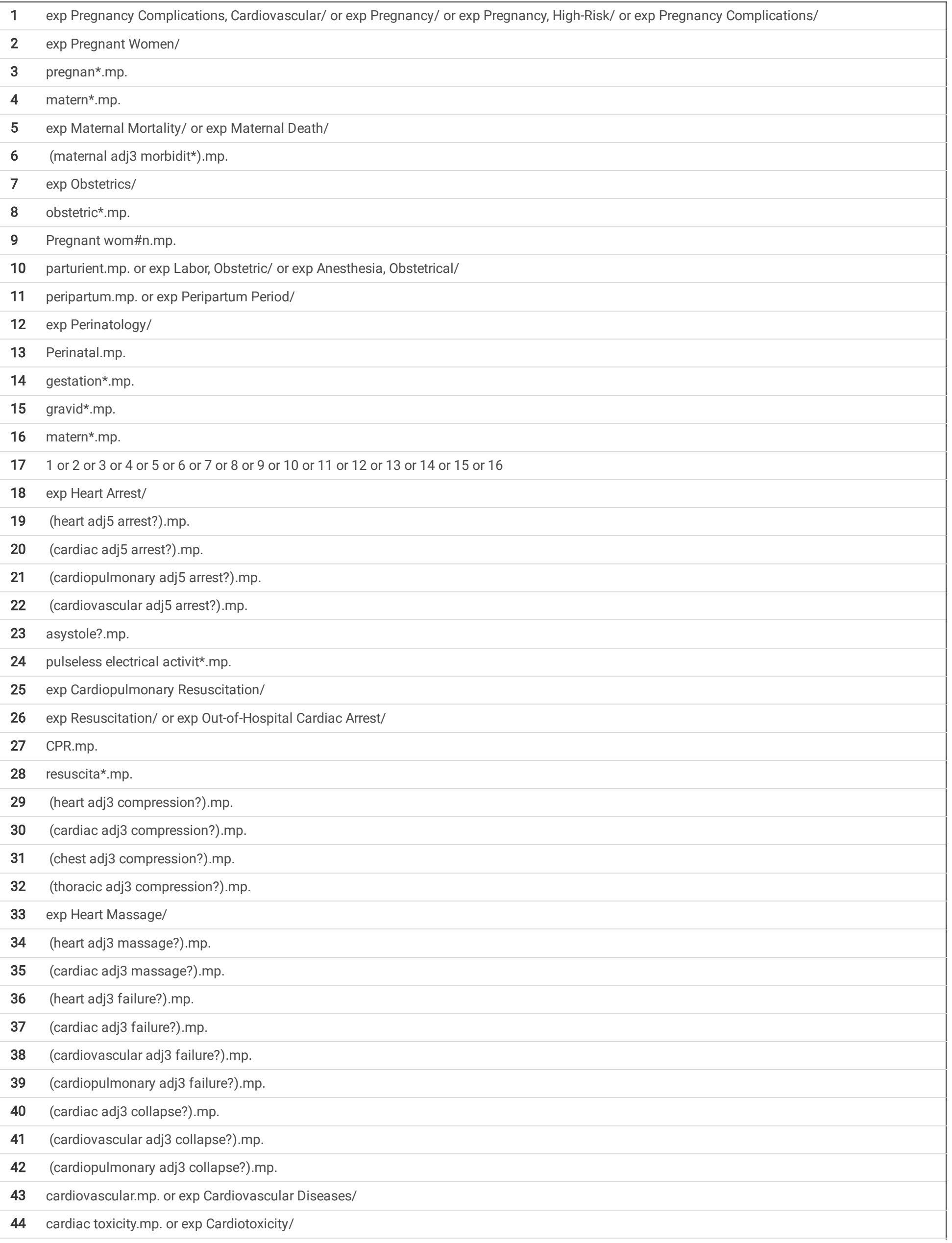




\begin{tabular}{|c|c|}
\hline 46 & (life adj3 support*).mp. \\
\hline 47 & emergency.mp. or exp Emergencies/ or exp Emergency Medical Services/ \\
\hline 48 & exp Ventricular Fibrillation/ \\
\hline 49 & electromechanical dissociation*.mp. \\
\hline 50 & AED.mp. \\
\hline 51 & $\begin{array}{l}18 \text { or } 19 \text { or } 20 \text { or } 21 \text { or } 22 \text { or } 23 \text { or } 24 \text { or } 25 \text { or } 26 \text { or } 27 \text { or } 28 \text { or } 29 \text { or } 30 \text { or } 31 \text { or } 32 \text { or } 33 \text { or } 34 \text { or } 35 \text { or } 36 \text { or } 37 \text { or } 38 \text { or } 39 \text { or } 40 \text { or } 41 \text { or } 42 \text { or } 43 \text { or } \\
44 \text { or } 45 \text { or } 46 \text { or } 48 \text { or } 50\end{array}$ \\
\hline 52 & uterine displacement.mp. \\
\hline 53 & (left adj5 table adj5 tilt).mp. \\
\hline 54 & tilt*.mp. \\
\hline 55 & (uter* adj5 displac*).mp. \\
\hline 56 & left-lateral.mp. \\
\hline 57 & (left adj3 lateral).mp. \\
\hline 58 & lateral tilt.mp. \\
\hline 59 & exp Patient Positioning/ \\
\hline 60 & Aortocaval compression.mp. \\
\hline 61 & (Aort* adj5 compression*).mp. \\
\hline 62 & 52 or 53 or 54 or 55 or 56 or 57 or 58 or 59 or 60 or 61 \\
\hline 63 & 17 and 51 and 62 \\
\hline 64 & limit 63 to humans \\
\hline
\end{tabular}

\section{Inclusion and Exclusion Criteria}

The study population included pregnant women who experienced cardiac arrest. We made no restrictions regarding maternal age, care settings or nationality. Regarding the intervention, we included studies that examined the effect of maternal positioning or methods to relieve aortocaval compression during CPR. We also included any type of study (randomised control trials [RCTs], nonrandomised clinical trials and observational studies). Because of the rarity of cardiac arrest during pregnancy, we included simulation-based studies using patient mannequins. We excluded reviews and commentaries as well as studies without English language abstracts.

\section{Outcomes of Interest}

The primary outcomes of interest included the survival rate of mothers or fetuses/neonates with favourable neurologic outcomes and the return of spontaneous circulation following maternal cardiac arrest. The secondary outcomes of interest were the quality of CPR and any adverse events.

\section{Study Selection}

We imported identified studies into Covidence, a web-based tool for systematic reviews. Two review authors (NE and TY) independently screened the studies for relevance based on titles and abstracts; they then screened based on full texts. We resolved any discrepancies via discussions with the review team until we reached a consensus.

\section{Data Extraction and Risk of Bias Assessment}

Using data extraction forms designed specifically for this review, two review authors (NE and MF) extracted data from the included studies. We contacted the authors of original studies to obtain missing information and unpublished data. Two review authors (MF and NE) independently assessed the risk of bias in the included studies using a revised Cochrane risk-of-bias tool (RoB2) developed specifically for crossover trials [28] because all studies included in this review applied a crossover design.

\section{Data Synthesis and Analysis}

Findings from nonrandomised crossover studies are presented narratively. Whenever sufficient data were available from RCTs to estimate the effect size of the intervention, we conducted meta-analyses using Cochrane's Review Manager (RevMan) version 5.3 [29]. We calculated the weighted mean difference and 
95\% confidence interval $(\mathrm{Cl})$ for continuous outcomes. We performed the random effects meta-analyses, because we assumed that the impact of the maternal positioning during cardiopulmonary resuscitation varied from study to study [30]. We assessed clinical heterogeneity (e.g. variability in the interventions such as chest compression on different surfaces; the floor or on the bed) as well as methodological heterogeneity (e.g. variability in study design such as RCTs or nonrandomised studies) within each comparison. Where meta-analyses were performed, we assessed statistical heterogeneity with tau ${ }^{2}$ in addition to visual inspection of the forest plots [31]. We conducted subgroup analyses based on the clinical heterogeneity (chest compression delivery surfaces). If there was a concern to the robustness of the result caused by missing outcome data, sensitivity analysis would have been performed, by comparing results from different methods of dealing with missing data (e.g. available case analysis, imputed case analysis) [32,33]

\section{Overall Quality of Evidence}

We used the Grading of Recommendations Assessment, Development and Evaluation (GRADE) approach [34] to assess the body of evidence for all the identified outcomes. We assigned one of four levels - high, moderate, low or very low - to each outcome by considering five domains, including the withinstudy risk of bias, inconsistency, indirectness, imprecision and publication bias [35]. If sufficient studies had been available (>=10), then we would have constructed funnel plots to assess publication bias.

\section{Results \\ Search Results}

The databases we searched identified 1,839 articles, including 346 duplicates. We screened a total of 1,490 titles and abstracts and selected 79 articles for full-text evaluation. We identified no additional articles from the reference lists of the included studies or review articles, and of the 79 articles that underwent full-text evaluation, we excluded 71 for the reasons stated in the PRISMA flowchart (Figure 1). A total of eight studies met the inclusion criteria, including six crossover RCTs [36-41] and two nonrandomised crossover studies [42,43].

\section{Characteristics of Included Studies}

An overview of included studies is presented in Table 2. We identified no studies that evaluated the effect of maternal positioning during CPR on primary outcomes; therefore, no data were available on survival rates and the return of spontaneous circulation following maternal cardiac arrest. Fetal/neonatal outcomes were also unavailable. The only outcomes available constituted indirect evidence of the quality of CPR, which was obtained from simulation-based studies using hypothetical cardiac arrest maternal patient mannequins. The studies originated from the United Kingdom $(n=4)$, South Korea $(n=2)$ and Japan ( $n=2)$. Of these, one randomised crossover study [39] examined the effect of the manual left uterine displacement in the supine position using a silicone pregnancy bump to simulate a woman in the third trimester of pregnancy and compared the results to those obtained in the left-lateral tilt achieved with a firm wedge. The remaining four randomised crossover studies [36-38,40] and one nonrandomised crossover study [42] compared the quality of chest compression on a mannequin lying supine with that of one lying on a $27^{\circ}-30^{\circ}$ left-inclined lateral tilting surface [36-38,40] or resting on the thighs of a kneeling assistant ('human wedge')[42]. One randomised crossover study examined the optimal methods for producing lateral tilt comparing soft pillow wedge: firm foam wedge, hard wooden wedge or human wedge [41], and one nonrandomised crossover study [43] examined the effect of chest compression at various angles between $0^{\circ}$ and $90^{\circ}$ of inclination. All participants in the included studies were health professionals (including medical students) and performed two or more sequential interventions. The quality of CPR was measured for chest compression (e.g. compression rate, depth, correct compression depth rate, correct recoil rates and/or correct hand position rate) and air ventilation. Subjective stability and difficulty (or ease) of chest compression also were assessed in some trials.

Table 2

Characteristics of included studies. 


\begin{tabular}{|c|c|c|c|c|c|c|}
\hline Authors/Year & Country & $\begin{array}{l}\text { Study } \\
\text { Design }\end{array}$ & Participants & Comparison & Device & Outcomes \\
\hline $\begin{array}{l}\text { Rees \& } \\
\text { Willis., } \\
1988 \text { [43] }\end{array}$ & UK & $\begin{array}{l}\text { Simulation } \\
\text { based non- } \\
\text { randomised } \\
\text { crossover } \\
\text { study }\end{array}$ & $\begin{array}{l}8 \text { medical doctors } \\
\text { (7 anaesthetists } \\
\text { and one } \\
\text { cardiologist) }\end{array}$ & $\begin{array}{l}\text { Chest compression (CC) in various } \\
\text { angles; } 0^{\circ}, 27^{\circ}, 32^{\circ}, 49^{\circ} \text { and } 90^{\circ} \text { left } \\
\text { lateral tilt (LTT) }\end{array}$ & $\begin{array}{l}\text { Calibrated force } \\
\text { transducer fitted } \\
\text { on the plane }\end{array}$ & $\begin{array}{l}\text { - Resuscitative } \\
\text { (compression) force } \\
\text { as \% of body weight } \\
\text { (mean, standard } \\
\text { deviation [SD]) }\end{array}$ \\
\hline $\begin{array}{l}\text { Goodwin } \\
1992[42]\end{array}$ & UK & $\begin{array}{l}\text { Simulation } \\
\text { based non- } \\
\text { randomised } \\
\text { crossover } \\
\text { study }\end{array}$ & 18 midwives & $\begin{array}{l}\text { CC in supine without manual left uterine } \\
\text { displacement (LUD) vs. CC in lateral tilt } \\
\text { produced by human wedge (the degree } \\
\text { of tilt not reported) }\end{array}$ & $\begin{array}{l}\text { Laerdal } \\
\text { Resusci Anne }{ }^{\circledR} \\
\text { Skill Reporting } \\
\text { System }\end{array}$ & $\begin{array}{l}\text { - Correct chest } \\
\text { compressions } \\
\text { (definition not } \\
\text { described), \% (mean, } \\
\text { SD) } \\
\text { - Correct expired air } \\
\text { ventilations } \\
\text { (definition not } \\
\text { described), (mean, } \\
\text { SD) }\end{array}$ \\
\hline \multirow[t]{7}{*}{$\begin{array}{l}\text { Lee et al. } \\
2011[36]\end{array}$} & \multirow[t]{7}{*}{$\begin{array}{l}\text { South } \\
\text { Korea }\end{array}$} & \multirow[t]{7}{*}{$\begin{array}{l}\text { Simulation- } \\
\text { based } \\
\text { crossover } \\
\text { RCT }\end{array}$} & \multirow[t]{7}{*}{$\begin{array}{l}30 \text { emergency } \\
\text { medical residents } \\
\text { and technicians }\end{array}$} & \multirow[t]{7}{*}{$\begin{array}{l}\text { CC in supine without manual LUD vs. CC } \\
\text { in } 30^{\circ} \text { LLT surface }\end{array}$} & $\begin{array}{l}\text { Laerdal } \\
\text { Resusci Anne }{ }^{\circledR}\end{array}$ & $\begin{array}{l}\text { - Compression rate, } \\
\text { per minute (mean, } \\
95 \% \text { confidence } \\
\text { interval [Cl]) }\end{array}$ \\
\hline & & & & & $\begin{array}{l}\text { Skill Reporting } \\
\text { System (Stavanger, } \\
\text { Norway) }\end{array}$ & $\begin{array}{l}\text { - Compression depth, } \\
\text { mm (mean, 95\% Cl) } \\
\text { - Correct } \\
\text { compression depth } \\
\text { rate, } 50-60 \mathrm{~mm} \% \\
\text { (mean, } 95 \% \mathrm{Cl} \text { ) }\end{array}$ \\
\hline & & & & & & $\begin{array}{l}\text { - Correct recoil rate, } \\
\%(\text { mean, } 95 \% \mathrm{Cl})\end{array}$ \\
\hline & & & & & & $\begin{array}{l}\text { - Correct hand } \\
\text { position rate, \% } \\
(\text { mean, } 95 \% \mathrm{Cl})\end{array}$ \\
\hline & & & & & & $\begin{array}{l}\text { - Highest } \\
\text { compression angle } \\
\text { (mean, } 95 \% \mathrm{Cl} \text { ) }\end{array}$ \\
\hline & & & & & & $\begin{array}{l}\text { - Lowest } \\
\text { compression angle } \\
\text { (mean, } 95 \% \mathrm{Cl} \text { ) }\end{array}$ \\
\hline & & & & & & $\begin{array}{l}\text { - Subjective difficulty } \\
\text { of CC, } 5 \text {-point Likert } \\
\text { scale (mean, 95\% Cl) }\end{array}$ \\
\hline \multirow{7}{*}{$\begin{array}{l}\text { Kim et al., } \\
2013 \\
{[37]}\end{array}$} & \multirow[t]{7}{*}{$\begin{array}{l}\text { South } \\
\text { Korea }\end{array}$} & \multirow{7}{*}{$\begin{array}{l}\text { Simulation- } \\
\text { based } \\
\text { crossover } \\
\text { RCT }\end{array}$} & \multirow{7}{*}{$\begin{array}{l}32 \text { BLS-trained } \\
\text { medical students } \\
\text { (inexperienced } \\
\text { rescuers without } \\
\text { CPR experience) }\end{array}$} & \multirow[t]{7}{*}{$\begin{array}{l}\text { CC in supine without manual LUD vs. CC } \\
\text { in } 30^{\circ} \text { LLT surface }\end{array}$} & \multirow{7}{*}{$\begin{array}{l}\text { Laerdal } \\
\text { Resusci } \\
\text { Anne }{ }^{\circledR} \text { Skill } \\
\text { Reporting System } \\
\text { (Stavanger, } \\
\text { Norway) }\end{array}$} & $\begin{array}{l}\text { - Compression rate, } \\
\text { per minute (mean, } \\
95 \% \mathrm{Cl} \text { ) }\end{array}$ \\
\hline & & & & & & $\begin{array}{l}\text { - Compression depth, } \\
\text { mm (mean, 95\% Cl) } \\
\text { - Correct } \\
\text { compression depth } \\
\text { rate, } 50-60 \mathrm{~mm} \% \\
\text { (mean, } 95 \% \mathrm{Cl} \text { ) }\end{array}$ \\
\hline & & & & & & $\begin{array}{l}\text { - Correct recoil rate, } \\
\%(\text { mean, } 95 \% \mathrm{Cl})\end{array}$ \\
\hline & & & & & & $\begin{array}{l}\text { - Correct hand } \\
\text { position rate, \% } \\
\text { (mean, } 95 \% \mathrm{Cl} \text { ) }\end{array}$ \\
\hline & & & & & & $\begin{array}{l}\text { - Highest } \\
\text { compression angle } \\
\text { (mean, } 95 \% \mathrm{Cl} \text { ) }\end{array}$ \\
\hline & & & & & & $\begin{array}{l}\text { - Lowest } \\
\text { compression angle } \\
\text { (mean, } 95 \% \mathrm{Cl} \text { ) }\end{array}$ \\
\hline & & & & & & $\begin{array}{l}\text { - Subjective difficulty } \\
\text { of CC, } 5 \text {-point Likert } \\
\text { scale (mean, } 95 \% \mathrm{Cl})\end{array}$ \\
\hline
\end{tabular}




\begin{tabular}{|c|c|c|c|c|c|c|}
\hline Authors/Year & Country & $\begin{array}{l}\text { Study } \\
\text { Design }\end{array}$ & Participants & Comparison & Device & Outcomes \\
\hline $\begin{array}{l}\text { Komasawa } \\
\text { et al., } \\
2013 \text { [38] }\end{array}$ & Japan & $\begin{array}{l}\text { Simulation- } \\
\text { based } \\
\text { crossover } \\
\text { RCT }\end{array}$ & $\begin{array}{l}27 \text { male medical } \\
\text { doctors (with CPR } \\
\text { experience) }\end{array}$ & $\begin{array}{l}\text { CC in supine without manual LUD vs. CC } \\
\text { in } 27^{\circ} \text { LLT surface } \\
\text { (by standing on the left and right } \\
\text { sides of the patient) }\end{array}$ & $\begin{array}{l}\text { Laerdal } \\
\text { Resuci Anne } \\
\text { Skill Reporting } \\
\text { System } \\
\text { (Stavanger, } \\
\text { Norway) }\end{array}$ & $\begin{array}{l}\text { - Compression rate, } \\
\text { per minute (mean, } \\
\text { SD) } \\
\text { - Compression depth } \\
\text { mm (mean, SD) } \\
\text { - Correct } \\
\text { compression depth } \\
\text { rate }(>50 \mathrm{~mm}), \% \\
\text { (mean, } 95 \% \mathrm{Cl} \text { ) } \\
\text { - Correct recoil rate, } \\
\% \text { (mean) }\end{array}$ \\
\hline $\begin{array}{l}\text { Ip et al. } \\
2013[41]\end{array}$ & UK & $\begin{array}{l}\text { Simulation- } \\
\text { based } \\
\text { crossover } \\
\text { RCT }\end{array}$ & $\begin{array}{l}40 \text { healthcare } \\
\text { professionals } \\
\text { (anaesthetists and } \\
\text { midwives) }\end{array}$ & $\begin{array}{l}\text { CC in the LLT with the soft wedge } \\
\text { (pillow) vs. firm wedge (foam-rubber) vs. } \\
\text { hard wedge (wooden) vs. human wedge }\end{array}$ & $\begin{array}{l}\text { Laerdal } \\
\text { Resusci } \\
\text { Anne }{ }^{\circledR} \text { Skill } \\
\text { Reporting System } \\
\text { (Kent, UK), }\end{array}$ & $\begin{array}{l}\text { - Compression rate, } \\
\text { per minute (mean, } \\
95 \% \mathrm{Cl} \text { ) } \\
\text { - Compression depth } \\
\text { mm (median, } \\
\text { interquartile range } \\
\text { [IQR]) } \\
\text { - Correct } \\
\text { compression depth } \\
\text { rate (> 50 mm), \% } \\
\text { (median, IQR) } \\
\text { - Correct recoil rate } \\
\text { (proportion of } \\
\text { compressions } \\
\text { adequately } \\
\text { released), \% (mean, } \\
\text { 95\% Cl) } \\
\text { - Subjective stability } \\
\text { of CC, 5-point Likert } \\
\text { scale (median, IQR) }\end{array}$ \\
\hline $\begin{array}{l}\text { Butcher et } \\
\text { al., } 2014 \text { [39] }\end{array}$ & UK & $\begin{array}{l}\text { Simulation- } \\
\text { based } \\
\text { crossover } \\
\text { RCT }\end{array}$ & $\begin{array}{l}20 \text { BLS/ALS-trained } \\
\text { healthcare } \\
\text { professionals } \\
\text { (10 anaesthetists } \\
\text { and } 10 \text { midwives) }\end{array}$ & $\begin{array}{l}\text { CC in supine with manual displacement } \\
\text { of uterus vs. CC in LLT produced by a } \\
\text { preformed firm-rubber wedge on the } \\
\text { floor and on a bed (angle not reported) }\end{array}$ & $\begin{array}{l}\text { Laerdal } \\
\text { Resusci } \\
\text { Anne }{ }^{\circledR} \text { Skill } \\
\text { Reporting System } \\
\text { (Kent, UK), with } \\
\text { 'pregnancy bump' }\end{array}$ & $\begin{array}{l}\text { - Compression rate, } \\
\text { per minute (mean, } \\
\text { SD) } \\
\text { - Compression depth } \\
\text { mm (median, IQR) } \\
\text { - Correct } \\
\text { compression depth } \\
\text { rate (> 50 mm; } \\
\text { median, IQR) } \\
\text { - Correct recoil rate, } \\
\% \text { (median, IQR) } \\
\text { - Subjective stability } \\
\text { and ease of CC, 5- } \\
\text { point Likert scale } \\
\text { (median, IQR) }\end{array}$ \\
\hline $\begin{array}{l}\text { Dohi et al., } \\
2017 \text { [40] }\end{array}$ & Japan & $\begin{array}{l}\text { Simulation- } \\
\text { based } \\
\text { crossover } \\
\text { RCT }\end{array}$ & $\begin{array}{l}20 \text { BLS-certified } \\
\text { healthcare } \\
\text { professionals }\end{array}$ & $\begin{array}{l}\text { CC in supine without manual LUD vs. } \\
30^{\circ} \text { LLT surface }\end{array}$ & $\begin{array}{l}\text { Laerdal } \\
\text { Skill Reporting } \\
\text { System }\end{array}$ & $\begin{array}{l}\text { - Compression rate, } \\
\text { per minute (mean, } \\
\text { SD) } \\
\text { - Compression depth } \\
\text { mm (mean, SD) } \\
\text { - Correct } \\
\text { compression depth } \\
\text { rate (50-60 mm), \% } \\
\text { (mean, SD) } \\
\text { - Correct recoil rate } \\
\text { (within } 5 \text { mm of } \\
\text { baseline chest } \\
\text { height), \% (mean, } \\
\text { SD) } \\
\text { - Correct hand } \\
\text { position rate, \% } \\
\text { (mean, SD) } \\
\text { - Subjective ease of } \\
\text { CC, } 5 \text {-point Likert } \\
\text { scale (mean, 95\%Cl) }\end{array}$ \\
\hline
\end{tabular}




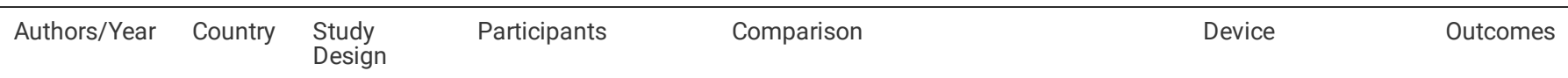

Abbreviations: RCT=Randomized Controlled Trial; $C C=$ chest compression; $L U D=$ left uterine displacement; $L L T=$ left lateral tilt; $C P R=$ cardiopulmonary resuscitation; $\mathrm{BLS}=$ Basic life support; $\mathrm{ALS}=$ advanced life support; $\mathrm{SD}=$ standard deviation; $\mathrm{Cl}=$ confidence interval; IQR=interquartile range.

\section{Risk of Bias Assessment}

\section{Bias due to randomisation.}

Of the randomised crossover trials included in this review [36-41],none except one [41] reported the processes used to generate the random allocation sequence and/or allocation concealment. Bias due to deviations from intended interventions: Given the nature of the interventions, participants (rescuers) in all studies were aware of their assigned intervention (e.g. chest compression in the supine or lateral tilting positions) during each period of the trial. Four studies $[36,37,40,41]$ ensured a washout period to minimise the carryover effect (after 2 minutes of chest compressions in the first assigned position, the participants rested for 10 minutes to minimise rescuer fatigue), whereas no information was available to assess the carryover effect in the remaining studies $[38,39,42,43]$. Bias due to missing outcome data: There were no missing outcomes [36-40,42,43], or the proportion of missing outcomes was small [41]. Bias due to outcome measurement. The outcomes were assessed using the PC SkillReporting software system, which was connected to the patient mannequin (Laerdal Resusci Anne ${ }^{\circledR}$ ) in all the included studies [36-42] except one [43]. Where the participants could not see the monitor screen displaying the outcomes during the chest compressions, the risk of bias was considered low [37,40]; however, where information regarding blinding of the outcomes was not provided, the risk of bias was rated as of some concern by taking into account the possibility that knowing the outcomes altered the participants' performance [36,38,39,41-43]. Bias due to selection of the reported result. No studies provided a trial protocol. Overall: Six RCTs [36-41] were rated as having some concern for risk bias, whereas the two nonrandomised crossover studies $[42,43]$ were considered of high risk for bias because at least one domain had a high risk of bias (Table 3).

Table 3

Risk of bias assessment (judgement and supporting evidence) in the included studies using a revised Cochrane risk-of-bias tool for crossover trials. 


\begin{tabular}{|c|c|c|c|c|c|c|}
\hline & $\begin{array}{l}\text { Bias due to } \\
\text { randomisation }\end{array}$ & $\begin{array}{l}\text { Bias due to } \\
\text { deviations from } \\
\text { intended } \\
\text { interventions }\end{array}$ & $\begin{array}{l}\text { Bias due to missing outcome } \\
\text { data }\end{array}$ & $\begin{array}{l}\text { Bias due to outcome } \\
\text { measurement }\end{array}$ & $\begin{array}{l}\text { Bias due to } \\
\text { selection of } \\
\text { the reported } \\
\text { result }\end{array}$ & Overall \\
\hline \multicolumn{7}{|l|}{ RCTs } \\
\hline $\begin{array}{l}\text { Butcher } \\
2014 \text { [39] }\end{array}$ & $\begin{array}{l}\text { Some concerns: Methods } \\
\text { used for randomisation } \\
\text { and allocation } \\
\text { concealment not stated }\end{array}$ & $\begin{array}{l}\text { Some concerns: } \\
\text { Washout period } \\
\text { to minimise the } \\
\text { carryover effect } \\
\text { not stated }\end{array}$ & $\begin{array}{l}\text { Low risk: Outcome data } \\
\text { available for all participants } \\
\text { randomised }\end{array}$ & $\begin{array}{l}\text { Some concerns: } \\
\text { Outcomes assessed } \\
\text { with a PC SkillReporting } \\
\text { system; blinding to the } \\
\text { assessor not reported }\end{array}$ & $\begin{array}{l}\text { Some } \\
\text { concerns: } \\
\text { Insufficient } \\
\text { information } \\
\text { available to } \\
\text { assess the } \\
\text { reporting bias }\end{array}$ & $\begin{array}{l}\text { Some } \\
\text { concerns }\end{array}$ \\
\hline $\begin{array}{l}\text { Dohi } \\
2017[40]\end{array}$ & $\begin{array}{l}\text { Some concerns: Methods } \\
\text { used for randomisation } \\
\text { and allocation } \\
\text { concealment not stated }\end{array}$ & $\begin{array}{l}\text { Low risk: 'Resting } \\
\text { for } 10 \text { min ... and } \\
\text { repeating CPR for } \\
2 \text { min in the } \\
\text { second assigned } \\
\text { position' }\end{array}$ & $\begin{array}{l}\text { Low risk: Outcome data } \\
\text { available for all participants } \\
\text { randomised }\end{array}$ & $\begin{array}{l}\text { Low risk: 'To avoid } \\
\text { potential bias, rescuers } \\
\text { were blinded to the } \\
\text { monitor screen } \\
\text { displaying compression } \\
\text { rate or hand position' }\end{array}$ & $\begin{array}{l}\text { Some } \\
\text { concerns: } \\
\text { Insufficient } \\
\text { information } \\
\text { available to } \\
\text { assess the } \\
\text { reporting bias }\end{array}$ & $\begin{array}{l}\text { Some } \\
\text { concerns }\end{array}$ \\
\hline $\begin{array}{l}\text { Ip } \\
2013[41]\end{array}$ & $\begin{array}{l}\text { Some } \\
\text { concerns: Randomisation } \\
\text { by drawing } \\
\text { lots/allocation } \\
\text { concealment not stated }\end{array}$ & $\begin{array}{l}\text { Low } \\
\text { risk: 'Sufficient } \\
\text { breaks between } \\
\text { tests' were taken } \\
\text { to minimise the } \\
\text { carryover effect }\end{array}$ & $\begin{array}{l}\text { Low risk: Outcome data } \\
\text { available for almost all } \\
\text { participants, although 'one of } \\
\text { the participants ... [was] } \\
\text { excluded from analysis due to } \\
\text { incomplete data capture' }\end{array}$ & $\begin{array}{l}\text { Some concerns: } \\
\text { Outcomes assessed } \\
\text { with a PC SkillReporting } \\
\text { system; blinding to the } \\
\text { assessor not reported }\end{array}$ & $\begin{array}{l}\text { Some } \\
\text { concerns: } \\
\text { Insufficient } \\
\text { information } \\
\text { available to } \\
\text { assess the } \\
\text { reporting bias }\end{array}$ & $\begin{array}{l}\text { Some } \\
\text { concerns }\end{array}$ \\
\hline $\begin{array}{l}\text { Kim } \\
2012 \text { [37] }\end{array}$ & $\begin{array}{l}\text { Some concerns: Methods } \\
\text { used for randomisation } \\
\text { and allocation } \\
\text { concealment not stated }\end{array}$ & $\begin{array}{l}\text { Low risk: 'The } \\
\text { participant rested } \\
\text { for } 10 \text { min to } \\
\text { minimise rescuer } \\
\text { fatigue' between } \\
\text { tests }\end{array}$ & $\begin{array}{l}\text { Low risk: Outcome data } \\
\text { available for all participants } \\
\text { randomised }\end{array}$ & $\begin{array}{l}\text { Low risk: 'To avoid } \\
\text { potential bias, } \\
\text { participants were } \\
\text { blinded to the monitor } \\
\text { screen' }\end{array}$ & $\begin{array}{l}\text { Some } \\
\text { concerns: } \\
\text { Insufficient } \\
\text { information } \\
\text { available to } \\
\text { assess the } \\
\text { reporting bias }\end{array}$ & $\begin{array}{l}\text { Some } \\
\text { concerns }\end{array}$ \\
\hline $\begin{array}{l}\text { Komasawa } \\
2013[38]\end{array}$ & $\begin{array}{l}\text { Some concerns: Methods } \\
\text { used for randomisation } \\
\text { and allocation } \\
\text { concealment not stated }\end{array}$ & $\begin{array}{l}\text { Some concerns: } \\
\text { Washout period } \\
\text { to minimise the } \\
\text { carryover effect } \\
\text { not stated }\end{array}$ & $\begin{array}{l}\text { Low risk: Outcome data } \\
\text { available for all participants } \\
\text { randomised }\end{array}$ & $\begin{array}{l}\text { Some concerns: } \\
\text { Outcomes assessed } \\
\text { with a PC SkillReporting } \\
\text { system; blinding to the } \\
\text { assessor not reported }\end{array}$ & $\begin{array}{l}\text { Some } \\
\text { concerns: } \\
\text { Insufficient } \\
\text { information } \\
\text { available to } \\
\text { assess the } \\
\text { reporting bias }\end{array}$ & $\begin{array}{l}\text { Some } \\
\text { concerns }\end{array}$ \\
\hline $\begin{array}{l}\text { Lee } \\
2011[36]\end{array}$ & $\begin{array}{l}\text { Some concerns: Methods } \\
\text { used for randomisation } \\
\text { and allocation } \\
\text { concealment not stated }\end{array}$ & $\begin{array}{l}\text { Some concerns: } \\
\text { Washout period } \\
\text { to minimise the } \\
\text { carryover effect } \\
\text { not stated }\end{array}$ & $\begin{array}{l}\text { Low risk: Outcome data } \\
\text { available for all participants } \\
\text { randomised }\end{array}$ & $\begin{array}{l}\text { Some concerns: } \\
\text { Outcomes assessed } \\
\text { with a PC SkillReporting } \\
\text { system; blinding to the } \\
\text { assessor not reported }\end{array}$ & $\begin{array}{l}\text { Some } \\
\text { concerns: } \\
\text { Insufficient } \\
\text { information } \\
\text { available to } \\
\text { assess } \\
\text { reporting bias }\end{array}$ & $\begin{array}{l}\text { Some } \\
\text { concerns }\end{array}$ \\
\hline \multicolumn{7}{|l|}{ Non-RCTs } \\
\hline $\begin{array}{l}\text { Goodwin } \\
1992[42]\end{array}$ & $\begin{array}{l}\text { High risk: } \\
\text { Not stated as being } \\
\text { randomised }\end{array}$ & $\begin{array}{l}\text { Some concerns: } \\
\text { Washout period } \\
\text { to minimise the } \\
\text { carryover effect } \\
\text { not stated }\end{array}$ & $\begin{array}{l}\text { Low risk: Outcome data } \\
\text { available for all participants } \\
\text { randomised }\end{array}$ & $\begin{array}{l}\text { Some concerns: } \\
\text { Outcomes assessed } \\
\text { with a PC SkillReporting } \\
\text { system; blinding to the } \\
\text { assessor not reported }\end{array}$ & $\begin{array}{l}\text { Some } \\
\text { concerns: } \\
\text { Insufficient } \\
\text { information } \\
\text { available to } \\
\text { assess the } \\
\text { reporting bias }\end{array}$ & $\begin{array}{l}\text { High } \\
\text { risk }\end{array}$ \\
\hline $\begin{array}{l}\text { Rees and } \\
\text { Wills } \\
1988 \text { [43] }\end{array}$ & $\begin{array}{l}\text { High risk: } \\
\text { Not stated as being } \\
\text { randomised }\end{array}$ & $\begin{array}{l}\text { Some concerns: } \\
\text { Washout period } \\
\text { to minimise the } \\
\text { carryover effect } \\
\text { not stated }\end{array}$ & $\begin{array}{l}\text { Low risk: Outcome data } \\
\text { available for all participants } \\
\text { randomised }\end{array}$ & $\begin{array}{l}\text { Some concerns: } \\
\text { Outcomes assessed } \\
\text { with a transducer; } \\
\text { blinding to the assessor } \\
\text { not reported }\end{array}$ & $\begin{array}{l}\text { Some } \\
\text { concerns: } \\
\text { Insufficient } \\
\text { information } \\
\text { available to } \\
\text { assess the } \\
\text { reporting bias }\end{array}$ & High risk \\
\hline
\end{tabular}

\section{Intervention Effectiveness}

\section{Maternal and Fetal (or Neonatal) Outcomes}

Because all the studies included in this review were conducted on mannequins, no data regarding maternal or fetal/neonatal outcomes were available for our analysis.

\section{Quality of CPR and Subjective Stability/Difficulty of Chest Compression}


All eight studies included in this review provided data regarding the quality of the CPR, and some provided data on subjective stability or difficulty (or ease) of chest compression (Table 4).

Table 4

Quality of CPR and subjective stability/difficulty of chest compression (Cont.).

\section{Comparison 1: Left lateral tilt position vs. manual left uterine displacement Quality of Chest Compression}

Based on one crossover RCT [39] involving 20 health professionals, there was no statistically significant difference in the quality of chest compressions as measured with compression rates, compression depth, correct compression depth (> $50 \mathrm{~mm}$ ) rates and correct recoil rates between the manual left uterine displacement in the supine position and the left lateral tilt position produced by a firm-rubber wedge. The results were consistent both on the floor and on the bed. The mean compression rates observed ranged from $114.5 / \mathrm{min}$ to $118.5 / \mathrm{min}$ and were within the range of adequate compression rates recommended by clinical guidelines. However, insufficient compression depth (median ranging from 40 to $44 \mathrm{~cm}$ ) and low rates of correct compression depth (median ranging from $25 \%$ to $57 \%$ ) were observed across all groups, indicating generally poor performance of chest compressions in the sample of this study.

\section{Subjective Ease and Stability of Chest Compression}

One study [39] involving inexperienced rescuers reported greater ease and stability of chest compressions in the supine position with manual left uterine displacement than in the left-lateral tilt position; the differences were statistically significant.

\section{Comparison 2: Left lateral tilt position $\left(\underline{27^{\circ}-30^{\circ}}\right)$ vs. supine position without manual left uterine displacement}

\section{Quality of Chest Compression}

A total of five studies including four crossover RCTs [36-38,40] and one nonrandomised crossover study [42] provided data on this outcome. Due to the methodological heterogeneity (i.e. RCTs or nonrandomised studies), only RCTs were included in the meta-analyses and results from nonrandomised study were presented separately in narrative form. 


\begin{tabular}{|c|c|c|c|c|c|c|c|c|c|c|c|c|}
\hline \multirow[t]{3}{*}{ Study } & \multirow[t]{3}{*}{ Design } & \multirow{3}{*}{$\begin{array}{l}\text { Comparison groups } \\
\text { (Chest compression } \\
\text { [CC] from right/left } \\
\text { side of patients) }\end{array}$} & \multirow{3}{*}{$\begin{array}{l}\text { Floor/ } \\
\text { Bed }\end{array}$} & \multirow[t]{3}{*}{$N$} & \multicolumn{3}{|c|}{ Correct hand position rate; $\%$} & \multicolumn{3}{|c|}{$\begin{array}{l}\text { Resuscitation } \\
\text { (compression) force; \% }\end{array}$} & \multicolumn{2}{|c|}{ Correct chest compressio } \\
\hline & & & & & \multicolumn{2}{|c|}{ Mean $(\mathrm{SD}$ or $95 \% \mathrm{Cl})$} & \multirow[t]{2}{*}{$P$} & \multicolumn{2}{|c|}{ Mean (SD or $95 \% \mathrm{Cl}$ ) } & \multirow[t]{2}{*}{$P$} & \multicolumn{2}{|c|}{ Mean (SD or $95 \% \mathrm{Cl})$} \\
\hline & & & & & $\begin{array}{l}\text { Left } \\
\text { lateral tilt }\end{array}$ & Supine & & $\begin{array}{l}\text { Left } \\
\text { lateral } \\
\text { tilt }\end{array}$ & Supine & & $\begin{array}{l}\text { Left } \\
\text { lateral } \\
\text { tilt }\end{array}$ & Supine \\
\hline
\end{tabular}

Comparison 1: Left lateral tilt position (LLT) vs. supine position with manual left uterine displacement (LUD)

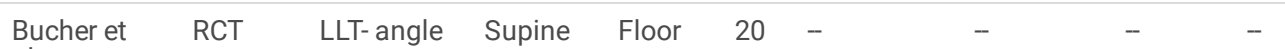

$\begin{array}{lll}\text { al. } & \text { not [39] } & \begin{array}{l}\text { reported } \\ \text { (right) }\end{array}\end{array}$

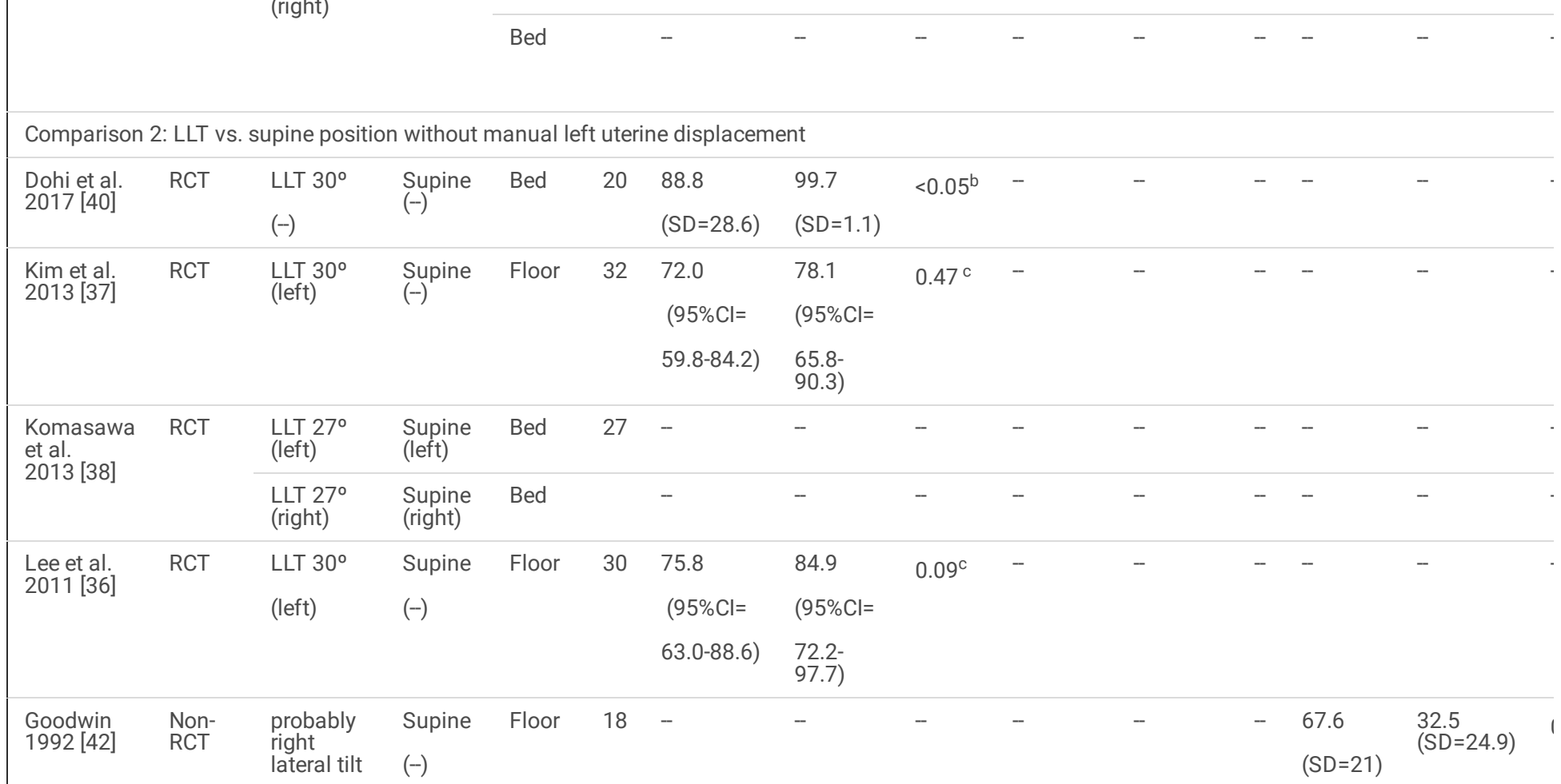

Comparison 3: Methods for producing LLT (soft wedge vs. firm wedge vs. hard wedge vs. human wedge)

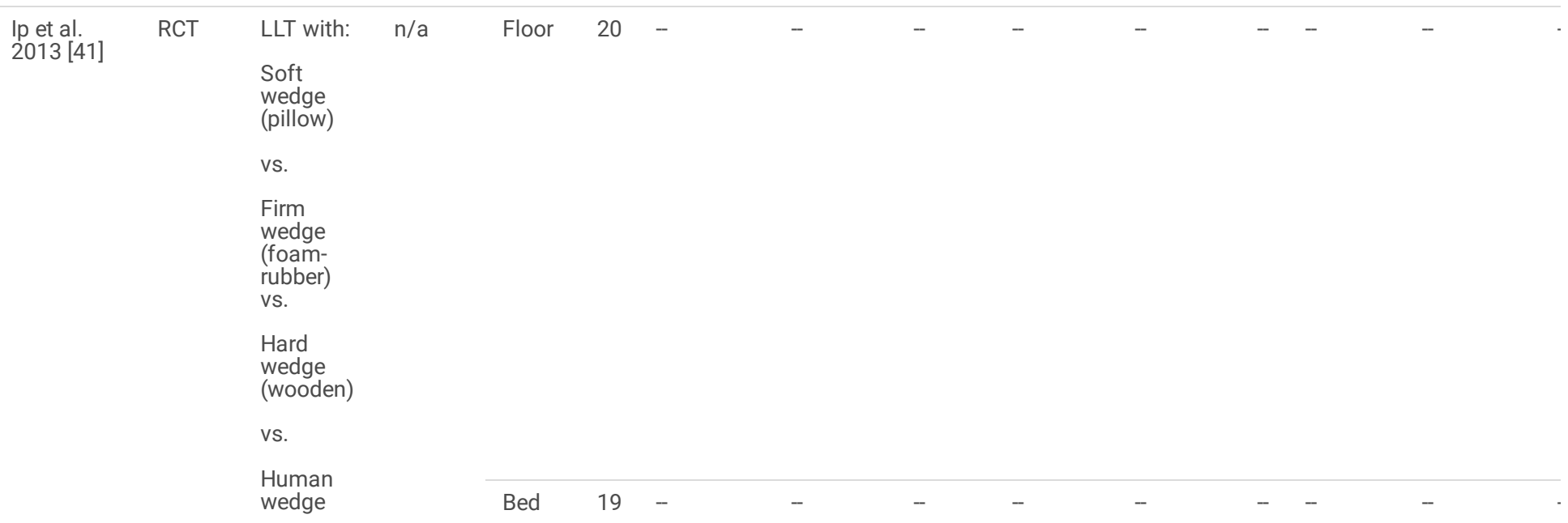


Comparison 4: Various angles $\left(0^{\circ}, 27^{\circ}, 32^{\circ}, 49^{\circ}\right.$ and $\left.90^{\circ}\right)$ of inclination

\begin{tabular}{|c|c|c|c|c|c|c|c|c|c|c|c|c|c|}
\hline \multirow[t]{7}{*}{$\begin{array}{l}\text { Rees \& } \\
\text { Willis } \\
1988 \text { [43] }\end{array}$} & \multirow[t]{7}{*}{$\begin{array}{l}\text { Non- } \\
\text { RCT }\end{array}$} & \multirow[t]{7}{*}{$\begin{array}{l}\text { LLT } 27^{\circ}, \\
32^{\circ}, 49^{\circ} \\
\text { and } 90^{\circ} \\
\text { (left) }\end{array}$} & \multirow[t]{7}{*}{$\begin{array}{l}\text { Supine } \\
(-)\end{array}$} & \multirow[t]{7}{*}{ Floor } & \multirow[t]{7}{*}{7} & \multirow[t]{7}{*}{-} & \multirow[t]{7}{*}{ - } & \multirow[t]{7}{*}{-} & $\begin{array}{l}\text { LLT 270: } \\
55.3 \\
(S D=5.5)\end{array}$ & \multirow[t]{7}{*}{$\begin{array}{l}66.7 \\
(S D=6.5)\end{array}$} & \multirow[t]{7}{*}{-} & \multirow[t]{7}{*}{-} & \multirow[t]{7}{*}{ - } \\
\hline & & & & & & & & & $\begin{array}{l}\text { LLT } 32^{\circ}: \\
46.4\end{array}$ & & & & \\
\hline & & & & & & & & & $(\mathrm{SD}=3.9)$ & & & & \\
\hline & & & & & & & & & $\begin{array}{l}\text { LLT 49o: } \\
41.5\end{array}$ & & & & \\
\hline & & & & & & & & & $(S D=3.5)$ & & & & \\
\hline & & & & & & & & & $\begin{array}{l}\text { LLT } 90^{\circ}: \\
36.3\end{array}$ & & & & \\
\hline & & & & & & & & & $(S D=5.4)$ & & & & \\
\hline
\end{tabular}

The four RCTs consistently showed no statistically significant differences between the supine and the left-lateral tilt position groups. Of these RCTs, one [38] was excluded from the meta-analysis (Figure 2) because of insufficient data provided in the original study (the means and standard deviations were unreported).

A total of four RCTs [36-38,40] assessed the rate of correct chest compression depth. The meta-analysis of these RCTs revealed the mean percentage of correct chest compression depth decreased by $17.25 \%$ when the left-lateral tilt position was used instead of the supine position; the difference was statistically significant (four RCTs, mean difference $[\mathrm{MD}]=-18.77,95 \% \mathrm{Cl}=-28.89,-8.64, \operatorname{tau}^{2}=48.95, \mathrm{I}^{2}=47 \%$; Figure 3 ). Subgroup analyses stratified by chest compression delivery surfaces (floor or bed) resulted in similar findings.

In addition, a meta-analysis of three RCTs [36-38] including a total of 89 health professionals revealed the mean chest compression depth was 2.88 mm lower in the $27^{\circ}-30^{\circ}$ left-lateral tilt position than in the supine position; the difference was statistically significant (three $\mathrm{RCTs}, \mathrm{MD}=-2.88 \mathrm{~mm}, 95 \% \mathrm{Cl}=-4.19,-1.57$, $\operatorname{tau}^{2}=0,1^{2}=0 \%$; Figure 4). The results were consistent across subgroups defined by the surface (floor or bed).

A total of four RCTs $[36-38,40]$ reported the recoil rates, none of which indicated statistically significant differences between the supine and the left-lateral tilt groups, either on the floor or on the bed.

A total of three RCTs $[36,37,40]$ reported the rate of correct hand positioning during chest compressions. The results of the meta-analysis indicated the correct hand position rate was $9 \%$ lower with the patient mannequin in the left-lateral tilt position than with it in the supine position (three RCTs, $\mathrm{MD}=-9.14,95 \% \mathrm{Cl}=$ $-17.8,-0.48, \operatorname{tau}^{2}=0,1^{2}=0 \%$; Figure 5).

There was one non-randomised crossover study conducted in 1992 [42] which reported that chest compression was significantly better (with the mean percentage of correct cardiac compression being approximately $34 \%$ higher) in the wedged position than in the supine position. The common reason for inaccuracy was 'compression of too great a force' (p. 434), but the study described neither the definition of correct cardiac compression nor the amount of compression force. The result was inconsistent from RCTs included in this review all of which were conducted more recently, in the 2010 s. The inconsistency could possibly due to both clinical and methodological heterogeneity - i.e. the difference in the outcome measures and study design (RCTs vs. nonrandomised crossover study) reflecting the time when studies were conducted.

\section{Subjective Difficulty (or Ease) of Chest Compressions}

Two RCTs $[37,40]$ involving both experienced and inexperienced rescuers reported that performing chest compressions in the left-lateral tilt position was significantly more difficult than doing so in the supine position, whereas another RCT [36] including only experienced emergency medical doctors reported no difference in the subjective difficulty between the two positions.

\section{Quality of Ventilation}

One nonrandomised crossover study [42] involving 18 midwives reported there was no statistically significate difference in the percentage of correct expired air ventilations (during performance of mouth-to-mouth resuscitation) between the supine and the left literal tilt positions (mean [SD] = 62.2\% [21.4] in the supine vs. $56.7 \%$ [27.7] in the left literal tilt positions). However, the definition of correct expired air ventilations was not described in the original study; it noted only that the commonest course of inaccurate ventilation was the ventilation of small volume.

\section{Comparison 3: Methods for producing left lateral tilt position (soft vs. firm vs. hard vs. human wedge).}




\begin{tabular}{|c|c|c|c|c|c|c|c|c|c|c|c|c|}
\hline \multirow[t]{3}{*}{ Study } & \multirow[t]{3}{*}{ Design } & \multirow{3}{*}{\multicolumn{2}{|c|}{$\begin{array}{l}\text { Comparison groups } \\
\text { (Chest compression } \\
\text { [CC] from right/left } \\
\text { side of patients) }\end{array}$}} & \multirow{3}{*}{$\begin{array}{l}\text { Floor/ } \\
\text { Bed }\end{array}$} & \multirow[t]{3}{*}{$\mathrm{N}$} & \multicolumn{3}{|c|}{ Subjective difficulty of CC } & \multicolumn{3}{|c|}{ Subjective ease of CC } & \multirow{2}{*}{$\begin{array}{l}\text { Correct expire } \\
\text { Mean (SD or } 9\end{array}$} \\
\hline & & & & & & \multicolumn{2}{|c|}{$\begin{array}{l}\text { Mean (SD or } \\
95 \% \mathrm{Cl})\end{array}$} & \multirow[t]{2}{*}{$P$} & \multicolumn{2}{|c|}{ Mean (SD or $95 \% \mathrm{Cl})$} & \multirow[t]{2}{*}{$P$} & \\
\hline & & & & & & $\begin{array}{l}\text { Left } \\
\text { lateral } \\
\text { tilt }\end{array}$ & Supine & & $\begin{array}{l}\text { Left lateral } \\
\text { tilt }\end{array}$ & Supine & & $\begin{array}{l}\text { Left } \\
\text { lateral tilt }\end{array}$ \\
\hline \multicolumn{13}{|c|}{ Comparison 1: Left lateral tilt position (LLT) vs. supine position with manual left uterine displacement (LUD) } \\
\hline \multirow[t]{2}{*}{$\begin{array}{l}\text { Bucher et } \\
\text { al. } 2014 \\
\text { [39] }\end{array}$} & \multirow[t]{2}{*}{ RCT } & \multirow{2}{*}{$\begin{array}{l}\text { LLT- angle } \\
\text { not } \\
\text { reported } \\
\text { (right) }\end{array}$} & \multirow[t]{2}{*}{$\begin{array}{l}\text { Supine } \\
\text { (right) }\end{array}$} & Floor & 20 & - & - & - & $\begin{array}{l}\text { median }=3.0 * \\
(\mathrm{IQR}=3-4)\end{array}$ & $\begin{array}{l}\text { median }=4.0 * \\
(\mathrm{IQR}=4-4)^{*}\end{array}$ & $0.011^{a}$ & - \\
\hline & & & & Bed & & - & - & - & $\begin{array}{l}\text { median }=4.0^{*} \\
(\mathrm{IQR}=3-4)\end{array}$ & $\begin{array}{l}\text { median }=5.0 \\
(\mathrm{IQR}=4-5)\end{array}$ & NS ${ }^{a}$ & - \\
\hline \multicolumn{13}{|c|}{ Comparison 2: LLT vs. supine position without manual left uterine displacement } \\
\hline \multirow{3}{*}{$\begin{array}{l}\text { Dohi et al. } \\
2017 \text { [40] }\end{array}$} & \multirow[t]{3}{*}{ RCT } & LLT $30^{\circ}$ & $\begin{array}{l}\text { Supine } \\
(-)\end{array}$ & Bed & 20 & - & - & - & $3.95^{\dagger}$ & $1.75^{\dagger}$ & $<0.001^{b}$ & - \\
\hline & & $(-)$ & & & & & & & $(95 \% \mathrm{Cl}=$ & $(95 \% \mathrm{Cl}=$ & & \\
\hline & & & & & & & & & $3.68-4.22)$ & $1.31-2.19)$ & & \\
\hline $\begin{array}{l}\text { Kim et al. } \\
2013 \text { [37] }\end{array}$ & $\mathrm{RCT}$ & $\begin{array}{l}\operatorname{LLT} 30^{\circ} \\
\text { (left) }\end{array}$ & $\begin{array}{l}\text { Supine } \\
(-)\end{array}$ & Floor & 32 & $68.8^{\ddagger}$ & $58.3^{\ddagger}$ & $0.007^{\mathrm{c}}$ & - & - & - & - \\
\hline & & & & & & $(95 \% \mathrm{Cl}=$ & $(95 \% \mathrm{Cl}=$ & & & & & \\
\hline & & & & & & $\begin{array}{l}62.8- \\
74.9)\end{array}$ & $\begin{array}{l}52.2- \\
64.4)\end{array}$ & & & & & \\
\hline Komasawa & $\mathrm{RCT}$ & $\begin{array}{l}\text { LLT } 27^{\circ} \\
\text { (left) }\end{array}$ & $\begin{array}{l}\text { Supine } \\
\text { (left) }\end{array}$ & Bed & 27 & - & - & - & - & - & - & - \\
\hline 2010 [0] & & $\begin{array}{l}\text { LLT } 27^{\circ} \\
\text { (right) }\end{array}$ & $\begin{array}{l}\text { Supine } \\
\text { (right) }\end{array}$ & Bed & & - & - & - & - & - & - & - \\
\hline $\begin{array}{l}\text { Lee et al. } \\
2011 \text { [36] }\end{array}$ & $\mathrm{RCT}$ & LLT $30^{\circ}$ & Supine & Floor & 30 & $68.4^{\ddagger}$ & $64.4^{\ddagger}$ & $0.28^{c}$ & - & - & - & - \\
\hline & & (left) & $(-)$ & & & $(95 \% \mathrm{Cl}=$ & $(95 \% \mathrm{Cl}=$ & & & & & \\
\hline & & & & & & $\begin{array}{l}62.1- \\
74.8)\end{array}$ & $\begin{array}{l}58.2- \\
71.0)\end{array}$ & & & & & \\
\hline $\begin{array}{l}\text { Goodwin } \\
1992[42]\end{array}$ & $\begin{array}{l}\text { Non- } \\
\text { RCT }\end{array}$ & $\begin{array}{l}\text { probably } \\
\text { right } \\
\text { lateral tilt }\end{array}$ & $\begin{array}{l}\text { Supine } \\
(-)\end{array}$ & Floor & 18 & - & - & - & - & - & - & $\begin{array}{l}56.7 \\
(S D=27.7)\end{array}$ \\
\hline Comparison & : Method & for producir & LLT (sof & wedge $v$ & firm & vedge vs. & ard wedge & s. humar & wedge) & & & \\
\hline Ip et al. & $\mathrm{RCT}$ & LLT with: & $\mathrm{n} / \mathrm{a}$ & Floor & 20 & - & - & - & - & - & - & - \\
\hline & & $\begin{array}{l}\text { Soft } \\
\text { wedge } \\
\text { (pillow) }\end{array}$ & & & & & & & & & & \\
\hline & & vs. & & & & & & & & & & \\
\hline & & $\begin{array}{l}\text { Firm } \\
\text { wedge } \\
\text { (foam- }\end{array}$ & & & & & & & & & & \\
\hline & & $\begin{array}{l}\text { rubber) } \\
\text { vs. }\end{array}$ & & Bed & 19 & - & - & - & - & - & - & - \\
\hline & & $\begin{array}{l}\text { Hard } \\
\text { wedge } \\
\text { (wooden) }\end{array}$ & & & & & & & & & & \\
\hline & & vs. & & & & & & & & & & \\
\hline & & $\begin{array}{l}\text { Human } \\
\text { wedge }\end{array}$ & & & & & & & & & & \\
\hline Comparison & 4: Various & angles $\left(0^{\circ}, 2\right.$ & $32^{\circ}, 49^{\circ}$ & nd $90^{\circ}$ ) & $\mathrm{f}$ incl & hation & & & & & & \\
\hline $\begin{array}{l}\text { Rees \& } \\
\text { Willis } \\
1988 \text { [43] }\end{array}$ & $\begin{array}{l}\text { Non- } \\
\text { RCT }\end{array}$ & $\begin{array}{l}\text { LLT } 27^{\circ} \\
32^{\circ}, 49^{\circ} \\
\text { and } 90^{\circ} \\
\text { (left) }\end{array}$ & $\begin{array}{l}\text { Supine } \\
(-)\end{array}$ & Floor & 7 & - & - & - & - & - & - & - \\
\hline
\end{tabular}

Note: $C C=$ chest compression; LUD=left; LLT=left lateral tilt; NS=not significant.

For Dohi et al. 2017, data were obtained from the authors. 
Statistical analysis used in the original studies: a) Wilcoxon signed rank sum; b) Student's t-test; c) mixed model; d) two-way repeated ANOVA; d) repeated AN tests; unless specified, statistical tests used were not reported in the original studies.

* 5-point Likert-scale: 1 =extremely (or very) poor; $2=$ poor; $3=$ adequate; $4=$ good; $5=$ excellent or very good (higher score =better stability or easier)

† 5-point Likert-scale: 1 =very easy; $2=$ easy; 3 =normal; $4=$ difficult; $5=$ very difficult (higher score = more difficult)

‡ Visual analogue scale from $0 \mathrm{~mm}=$ extremely east to $100 \mathrm{~mm}=$ extremely difficult (higher score = more difficult)

\section{Quality of Chest Compression}

One crossover RCT reported that the type of wedge - the soft wedge (pillow), firm wedge (foam-rubber), hard wedge (wooden) or human wedge - had no effect on the average rate or adequate release of chest compressions. The study consistently indicated that the depth of compressions (compression depth $[\mathrm{mm}]$ and rate of correct compression depth $>50 \mathrm{~mm}$ ) was reduced with the human wedge compared with other wedges; the differences were statistically significant during chest compressions on the floor but not on the bed.

\section{Subjective Stability of Chest Compressions}

One crossover RCT reported that the firm and hard wedges were the most stable (stability rated as 'good' or 'very good'), whereas the soft wedges were the least stable during chest compressions during chest compression on either the floor or bed.

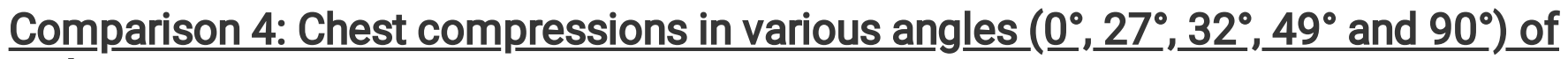 inclination}

\section{Quality of Chest Compression}

In one nonrandomised trial [43] involving eight medical doctors, the maximum possible resuscitative force (as measured with calibrated force transducer fitted on the plane) decreased as the angle of inclination of the plane increased, from $67 \%$ of body weight in the supine position to $36 \%$ in the full lateral.

\section{Quality of Evidence}

For all the outcomes included in this review related to the quality of CPR (chest compression and ventilation) and subjective stability or difficulty of chest compressions, the quality of evidence was rated as very low using the GRADE criteria and was downgraded for risk of bias (all evidence came from studies with some limitations for multiple domains), indirectness (simulation-based) and imprecision (wide Cls).

\section{Discussion}

Our systematic review evaluated the effect of maternal positioning for successful resuscitation of pregnant women. We identified no studies that evaluated the outcomes with real maternal patients. However, there were eight simulation-based crossover trials (six RCTs and two non-RCTs) that specifically examined the impact of maternal positioning or strategies on the quality of chest compression for hypothetical cardiac arrest maternal patients using a mannequin. Overall, meta-analyses of RCTs indicated resuscitation in the supine position enhances the quality of chest compressions by increasing the rates of correct compression depth and correct hand position, compared with resuscitation in the $27^{\circ}-30^{\circ}$ left lateral tilt position in pregnant women. The results were consistent for chest compressions performed both on the bed and on the floor. This review also suggested chest compressions in the left-lateral tilt position may be more difficult than chest compressions in the supine position for inexperienced health professionals.

\section{Comparison with Existing Guidelines and Reviews}

The 2020 American Heart Association (AHA) Guidelines [44] recommend that 'priorities for the pregnant woman in cardiac arrest should include provision of high-quality CPR and relief of aortocaval compression through left lateral uterine displacement' (Supplement, p. 454). This recommendation is based primarily on the physiology of pregnancy and extrapolations from nonarrest pregnancy states $[45,46]$ as well as nonrandomised simulation-based studies [42,43]. However, the interpretation of the recommendation is not straightforward because the recommendation was based on inconsistent results, including the nonrandomised simulation-based studies [42,43] that were conducted 30 to 40 years ago. Goodwin [42] reported that the quality of chest compression was reduced in the supine position compared to the wedged position using the human wedge manoeuvre. The common reason for inaccuracy was 'compression of too great a force' (p. 434). Our systematic review revealed that findings from recent RCTs contradict Goodwin's (1992) findings, possibly because of changes in the recommendations for CPR in past decades [47]. The current CPR guidelines recommend target chest compression depth in a victim's chest of 5- $6 \mathrm{~cm}$, whereas it was ' 4 to $5 \mathrm{~cm}$ ' (AHA Guidelines 2005) in the past; even further back, it was defined as the difference in the height of a rescuer's shoulder, not in a victim's chest, by 2.5 to $5 \mathrm{~cm}$ in the 1992 AHA Guidelines [48].

Although our review included only indirect evidence from simulation-based studies, the trials included had more sophisticated studies overcoming the methodological limitations commonly observed in previous studies (such as lack of randomisation, the potential risk of carryover effect and the inaccuracy of 
measuring outcomes). Our results suggested resuscitation in the supine position enhances the quality of resuscitation activity. Together with evidence from previous systematic reviews on the nonarrest pregnant population $[16,49]$ that suggested manual left uterine displacement effectively relieves aortocaval pressure in pregnant women with hypotension, it is reasonable to conclude that manual left uterine displacement in the supine position is more effective than a left lateral tilt position in increasing the quality of chest compression during resuscitation; in turn, this could contribute to increased maternal and fetal survival rates following maternal cardiac arrest.

\section{Strengths and Limitations of the Review}

Given its systematic and comprehensive literature search, our review enhanced the evidence regarding the effect of maternal positioning during maternal CPR, particularly on the quality of chest compressions. We translated a non-English study written in Korean because the authors' abstract was written in English and thus met our inclusion criteria. Where we found information in the included studies to be insufficient, we contacted the original researcher if doing so was possible. However, the quality of evidence produced by our systematic review was still low; this was mainly because of indirect evidence from the mannequin studies. From the study design point of view, all studies included in our review were crossover trials in which each healthcare professional involved performed chest compressions on a mannequin in two or more maternal positions in random order. Because each participant acted as their own control, this design allowed the participant to express the difficulty of chest compressions that they experienced for a particular maternal position. The crossover trials could have provided more precise estimates of the effect size than parallel-group trials if appropriate statistical analyses (paired analyses) had been applied [50,51], but this was not the case in some of our included studies. Data on within-subjects correlation were unavailable, so this advantage of a crossover design could not be utilised. Our meta-analysis estimated the average effect of intervention (maternal positioning), but given the small number of studies to be synthesised for each outcome, the statistical model used for meta-analysis (random effects model) had the limitation to estimate the between-study variance (the extent of variation among the effects observed in different studies).

\section{Further Research}

Knowledge gaps still exist concerning the effect and efficacy of CPR with manual uterine displacement versus tilt positioning on clinical outcomes following maternal cardiac arrest. Simulation-based RCTs specifically designed to evaluate the favourable or unfavourable effects of manual left uterine displacement should be carried out to assess the quality of CPR, including delay and interruption of CPR in relation to performing manual left uterine displacement. Further studies also must focus on establishing what could be the best strategies (including for manual left uterine displacement) for high-quality CPR. This is important because there are various recommendations regarding manual left uterine displacement, possibly referencing the situations or settings wherein maternal cardiac arrest occurs. For example, guidelines recommend 'placing a hand below the uterus on the maternal right and pushing the uterus slightly upwards and to the left' [46, p.29], which can be done with one-hand or two-hand techniques and from the left or right side of the patient [23,44,52]. There is, however, a lack of evidence about whether and how these different strategies affect the quality of CPR. Because maternal cardiac arrest is rare and RCTs evaluating the effects of maternal position with real patients would be unrealistic, the development of a nationwide database that collects data concerning both in-hospital and out-of-hospital maternal cardiac arrest patients would be beneficial. Such a database would be critical to predict the clinical outcomes of such cases, including the survival rates of mothers and babies with favourable neurologic outcomes, after cardiac arrest vis-à-vis the strategies used for relieving aortocaval compression during maternal resuscitation.

\section{Conclusion}

Although rare, cardiac arrest during pregnancy is the leading cause of maternal death. Recently, its incidence has been increasing worldwide because more pregnant women have risk factors. The provision of early, high-quality cardiopulmonary resuscitation (CPR) plays a major role in the increased likelihood of survival; therefore, clinicians should be familiar with its management. Due to the aortocaval compression caused by the gravid uterus, clinical guidelines often emphasise the importance of maternal positioning during CPR, but there has been little evidence regarding which position is most effective. Our systematic review synthesised evidence from trials published in recent years. Meta-analyses of simulation-based studies indicated that the resuscitation of pregnant women in the $27^{\circ}-30^{\circ}$ left-lateral tilt position lowered the quality of chest compressions compared with resuscitation in the supine position (the rates of correct compression depth and correct hand position decreased by about $17 \%$ and $9 \%$, respectively). Inexperienced clinicians faced difficulties in performing chest compressions in the left-lateral tilt position. Given that manual left uterine displacement allows the patient to remain supine, the resuscitation of women in the supine position using manual left uterine displacement should continue to be supported. Further research is needed to fill knowledge gaps regarding the effects of maternal positioning on clinical outcomes, such as survival rates following maternal cardiac arrest.

\section{Abbreviations}

ALS: advanced life support; BLS: Basic life support; CPR: cardiopulmonary resuscitation; LLT: left lateral tilt; LUD: left uterine displacement; RCT: Randomised Controlled Trial;

\section{Declarations}

\section{Ethics approval and consent to participate}

Not applicable 


\section{Consent for publication}

Not applicable

\section{Availability of data and materials}

The datasets used and/or analysed during the current study are available from the corresponding author on reasonable request.

\section{Competing interests}

The authors declare that they have no competing interests.

\section{Funding}

This research received no specific grant from any funding agency in the public, commercial, or not-for-profit sectors.

\section{Authors' contributions}

Drs Enomoto, Yamashita, Furuta, Tanaka, Matsunaga and Sakurai conceptualized and designed the study and developed search strategies. Drs Enomoto and Furuta designed the data extraction sheet. Drs Furuta and $\mathrm{Ng}$ extracted data analysed and interpreted data. Drs Enomoto, Yamashita, Furuta and Tanaka drafted the manuscript. Drs Matsunaga, Sakurai and $\mathrm{Ng}$ reviewed and revised the manuscript. All authors approved the final manuscript as submitted and agreed to be accountable for all aspects of the work.

\section{Acknowledgements}

The authors would like to thank the members of Japan Resuscitation Council, Maternal group; Dr. Rie Kato, Dr. Shinji Takahashi, Dr. Jun Takeda, Dr. Masahiro Nakao, Dr. Eishin Nakamura, Dr. Masafumi Nii, Dr. Yoshihiro Yamahata, Dr. Makoto Tsuji, Dr. Takahide Maenaka, Dr. Shinji Baba, and Dr. Yuki Hosokawa, members of Japan Resuscitation Council; Dr. Hiroshi Nonogi, Dr. Mayumi Aibiki, Dr. Takanari Ikeyama, Dr. Tetsuya Isayama, Dr. Taku Iwami, Dr. Yasuhiro Kuroda, Dr. Tetsuya Sakamoto, Dr. Naoki Shimizu, Dr. Masao Nagayama, Dr. Chika Nishiyama, Dr. Tetsuo Hatanaka, Dr. Shigeharu Hosono, Dr. Tasuku Matsuyama.

\section{References}

1. Briller J. Cardiopulmonary Resuscitation of Pregnant Women. In: Elkayam U, ed. Cardiac Problems in Pregnancy. Hoboken, NJ: Wiley Blackwell, 2020.

2. Hasegawa J, Sekizawa A, Tanaka H, et al. Current status of pregnancy-related maternal mortality in Japan: a report from the Maternal Death Exploratory Committee in Japan. BMJ Open 2016; 6: e010304.

3. Knight M, Clarke B, Head C, et al. Lessons on cardiovascular care. In: Knight M, Bunch K, Tuffnell D, et al., eds. Saving Lives, Improving Mothers' Care Lessons learned to inform maternity care from the UK and Ireland Confidential Enquiries into Maternal Deaths and Morbidity 2015-17. 0xford: National Perinatal Epidemiology Unit, University of Oxford, 2019: p20-44.

4. Balki M, Liu S, Leon JA, Baghirzada L. Epidemiology of cardiac arrest during hospitalization for delivery in Canada: a nationwide study. Anesthesia \& Analgesia 2017; 124: 890-7.

5. Beckett VA, Knight M, Sharpe P. The CAPS Study: incidence, management and outcomes of cardiac arrest in pregnancy in the UK: a prospective, descriptive study. BJOG: An International Journal of Obstetrics \& Gynaecology 2017; 124: 1374-81.

6. Mhyre JM, Tsen LC, Einav S, Kuklina EV, Leffert LR, Bateman BT. Cardiac arrest during hospitalization for delivery in the United States, $1998-2011$. Anesthesiology 2014; 120: 810-8.

7. Schaap TP, Overtoom E, van den Akker T, Zwart JJ, van Roosmalen J, Bloemenkamp KWM. Maternal cardiac arrest in the Netherlands: A nationwide surveillance study. European Journal of Obstetrics and Gynecology and Reproductive Biology 2019; 237: 145-50.

8. Jeejeebhoy F, Windrim R. Management of cardiac arrest in pregnancy. Best Practice and Research: Clinical Obstetrics and Gynaecology $2014 ; 28: 607-18$.

9. Tanaka H, Katsuragi S, Osato K, et al. The increase in the rate of maternal deaths related to cardiovascular disease in Japan from 1991-1992 to 20102012. J Cardiol 2017; 69: 74-8.

10. Kikuchi J, Deering S. Cardiac arrest in pregnancy. Seminars in Perinatology 2018; 42: 33-8.

11. Mehta LS, Warnes CA, Bradley E, et al. Cardiovascular Considerations in Caring for Pregnant Patients: A Scientific Statement From the American Heart Association. Circulation 2020; 141: e884-e903.

12. Zelop CM, Einav S, Mhyre JM, Martin S. Cardiac arrest during pregnancy: ongoing clinical conundrum. American Journal of Obstetrics and Gynecology 2018; 219: 52-61.

13. Lipowicz AA, Cheskes S, Gray SH, et al. Incidence, outcomes and guideline compliance of out-of-hospital maternal cardiac arrest resuscitations: A population-based cohort study. Resuscitation 2018; 132: 127-32.

Page $17 / 23$ 
14. Jeejeebhoy FM, Zelop CM, Lipman S, et al. Cardiac Arrest in Pregnancy: A Scientific Statement From the American Heart Association. Circulation 2015; 132: $1747-73$.

15. Helviz Y, Einav S. Maternal cardiac arrest. Current Opinion in Anaesthesiology 2019; 32: 298-306.

16. Cluver C, Novikova N, Hofmeyr GJ, Hall DR. Maternal position during caesarean section for preventing maternal and neonatal complications. Cochrane Database of Systematic Reviews 2013: CD007623.

17. Chesnutt AN. Physiology of normal pregnancy. Critical Care Clinics 2004; 20: 609-15.

18. Sanders AB, Meislin HW, Ewy GA. The physiology of cardiopulmonary resuscitation. An update. JAMA 1984; 252: 3283-6.

19. Chu J, Johnston TA, Geoghegan J, Royal College of O, Gynaecologists. Maternal Collapse in Pregnancy and the Puerperium: Green-top Guideline No. 56. BJOG: An International Journal of Obstetrics \& Gynaecology 2020; 127: e14-e52.

20. Kerr MG. The Mechanical Effects of the Gravid Uterus in Late Pregnancy. Journal of Obstetrics \& Gynaecology of the British Commonwealth 1965; 72: 513-29.

21. Gabbott DA. Uterine displacement during CPR in the pregnant patient-why bother? Resuscitation 2013; 84: $267-8$.

22. Lavonas EJ, Drennan IR, Gabrielli A, et al. Part 10: Special circumstances of resuscitation: 2015 American Heart Association guidelines update for cardiopulmonary resuscitation and emergency cardiovascular care. Circulation 2015; 132: S501-S18.

23. Lott C, Truhlar A, Alfonzo A, et al. European Resuscitation Council Guidelines 2021: Cardiac arrest in special circumstances. Resuscitation 2021; 161: 152-219.

24. Truhlar A, Deakin CD, Soar J, et al. European Resuscitation Council Guidelines for Resuscitation 2015: Sect. 4. Cardiac arrest in special circumstances. Resuscitation 2015; 95: 148-201.

25. Vanden Hoek TL, Morrison LJ, Shuster M, et al. Part 12: cardiac arrest in special situations: 2010 American Heart Association Guidelines for Cardiopulmonary Resuscitation and Emergency Cardiovascular Care. Circulation 2010; 122: S829-61.

26. Neumar RW, Shuster M, Callaway CW, et al. Part 1: Executive Summary. Circulation 2015; 132: S315-S67.

27. Moher D, Liberati A, Tetzlaff J, Altman DG, Group P. Preferred reporting items for systematic reviews and meta-analyses: the PRISMA statement. Journal of Clinical Epidemiology 2009; 62: 1006-12.

28. Sterne JAC, Savovic J, Page MJ, et al. RoB 2: a revised tool for assessing risk of bias in randomised trials. BMJ 2019; 366 : I4898.

29. Higgins JPT, Thomas J, Chandler J, et al. Cochrane Handbook for Systematic Reviews of Interventions. Chichester, UK: John Wiley \& Sons, 2019.

30. Borenstein M, Hedges LV, Higgins JPT, Rothstein H. Introduction to meta-analysis. Chichester, U.K: John Wiley \& Sons. Chichester, U.K: John Wiley \& Sons., 2009.

31. Deeks JJ, Higgins JPT, Altman DG, on behalf of the Cochrane Statistical Methods Group. Analysing data and undertaking meta-analyses. In: Higgins JPT, Thomas J, Chandler J, et al., eds. Cochrane Handbook for Systematic Reviews of Interventions. Chichester, UK: John Wiley \& Sons, 2019.

32. Gamble C, Hollis S. Uncertainty method improved on best-worst case analysis in a binary meta-analysis. J Clin Epidemio/ 2005; 58: 579-88.

33. Higgins JP, White IR, Wood AM. Imputation methods for missing outcome data in meta-analysis of clinical trials. Clin Trials 2008; 5: 225-39.

34. Guyatt GH, Oxman AD, Vist GE, et al. GRADE: an emerging consensus on rating quality of evidence and strength of recommendations. $B M J 2008$; 336 : 924-6.

35. Schünemann HJ, Higgins JPT, Vist GE, et al. Completing 'Summary of findings' tables and grading the certainty of the evidence. In: Higgins JPT, Thomas J, Chandler J, et al., eds. Cochrane Handbook for Systematic Reviews of Interventions. Chichester, UK: John Wiley \& Sons, 2019.

36. Lee JH, Choa M, Park JS, Kim S, Park YS, Chung SP. Comparative Evaluation of Chest Compression in a 30degrees Inclined Lateral Position Designed for Pregnant Cardiac Arrest Patients-Manikin Study. Journal of the korean society of emergency medicine 2011; 22: 650-5.

37. Kim S, You JS, Lee HS, et al. Quality of chest compressions performed by inexperienced rescuers in simulated cardiac arrest associated with pregnancy. Resuscitation 2013; 84: 98-102.

38. Komasawa N, Ueki R, Yamamoto N, Kaminoh Y, Tashiro C. Comparison of left-side and right-side approaches for chest compressions in the left-lateral tilt position: a manikin study of maternal cardiopulmonary resuscitation. International journal of obstetric anesthesia 2013; 22: 354-5.

39. Butcher M, Ip J, Bushby D, Yentis SM. Efficacy of cardiopulmonary resuscitation in the supine position with manual displacement of the uterus vs lateral tilt using a firm wedge: a manikin study. Anaesthesia 2014; 69: 868-71.

40. Dohi S, Ichizuka K, Matsuoka R, Seo K, Nagatsuka M, Sekizawa A. Coronary perfusion pressure and compression quality in maternal cardiopulmonary resuscitation in supine and left-lateral tilt positions: a prospective, crossover study using mannequins and swine models. European Journal of Obstetrics Gynecology and Reproductive Biology 2017; 216: 98-103.

41. Ip JK, Campbell JP, Bushby D, Yentis SM. Cardiopulmonary resuscitation in the pregnant patient: A manikin-based evaluation of methods for producing lateral tilt. Anaesthesia 2013; 68: 694-9.

42. Goodwin AP, Pearce AJ. The human wedge. A manoeuvre to relieve aortocaval compression during resuscitation in late pregnancy. Anaesthesia 1992; 47: 433-4.

43. Rees GA, Willis BA. Resuscitation in late pregnancy. Anaesthesia 1988; 43: 347-9.

44. Panchal AR, Bartos JA, Cabanas JG, et al. Part 3: Adult Basic and Advanced Life Support: 2020 American Heart Association Guidelines for Cardiopulmonary Resuscitation and Emergency Cardiovascular Care. Circulation 2020; 142: S366-S468.

45. Mendonca C, Griffiths J, Ateleanu B, Collis RE. Hypotension following combined spinal-epidural anaesthesia for Caesarean section. Left lateral position vs. tilted supine position. Anaesthesia 2003; 58: 428-31. 
46. Cyna AM, Andrew M, Emmett RS, Middleton P, Simmons SW. Techniques for preventing hypotension during spinal anaesthesia for caesarean section. Cochrane Database of Systematic Reviews 2006: CD002251.

47. Kwon OY. The changes in cardiopulmonary resuscitation guidelines: from 2000 to the present. J Exerc Rehabil 2019; 15: 738-46.

48. Guidelines for cardiopulmonary resuscitation and emergency cardiac care. Emergency Cardiac Care Committee and Subcommittees, American Heart Association. Part III. Adult advanced cardiac life support. JAMA 1992; 268: 2199-241.

49. Kundra P, Khanna S, Habeebullah S, Ravishankar M. Manual displacement of the uterus during Caesarean section. Anaesthesia 2007; 62: 460-5.

50. Elbourne DR, Altman DG, Higgins JP, Curtin F, Worthington HV, Vail A. Meta-analyses involving cross-over trials: methodological issues. International Journal of Epidemiology 2002; 31: 140-9.

51. Senn S. Cross-over trials in clinical research, 2nd edn. Chichester, Eng.; New York: J. Wiley, 2002.

52. Queensland Clinical Guidelines. Trauma in pregnancy, 2019.

\section{Figures}

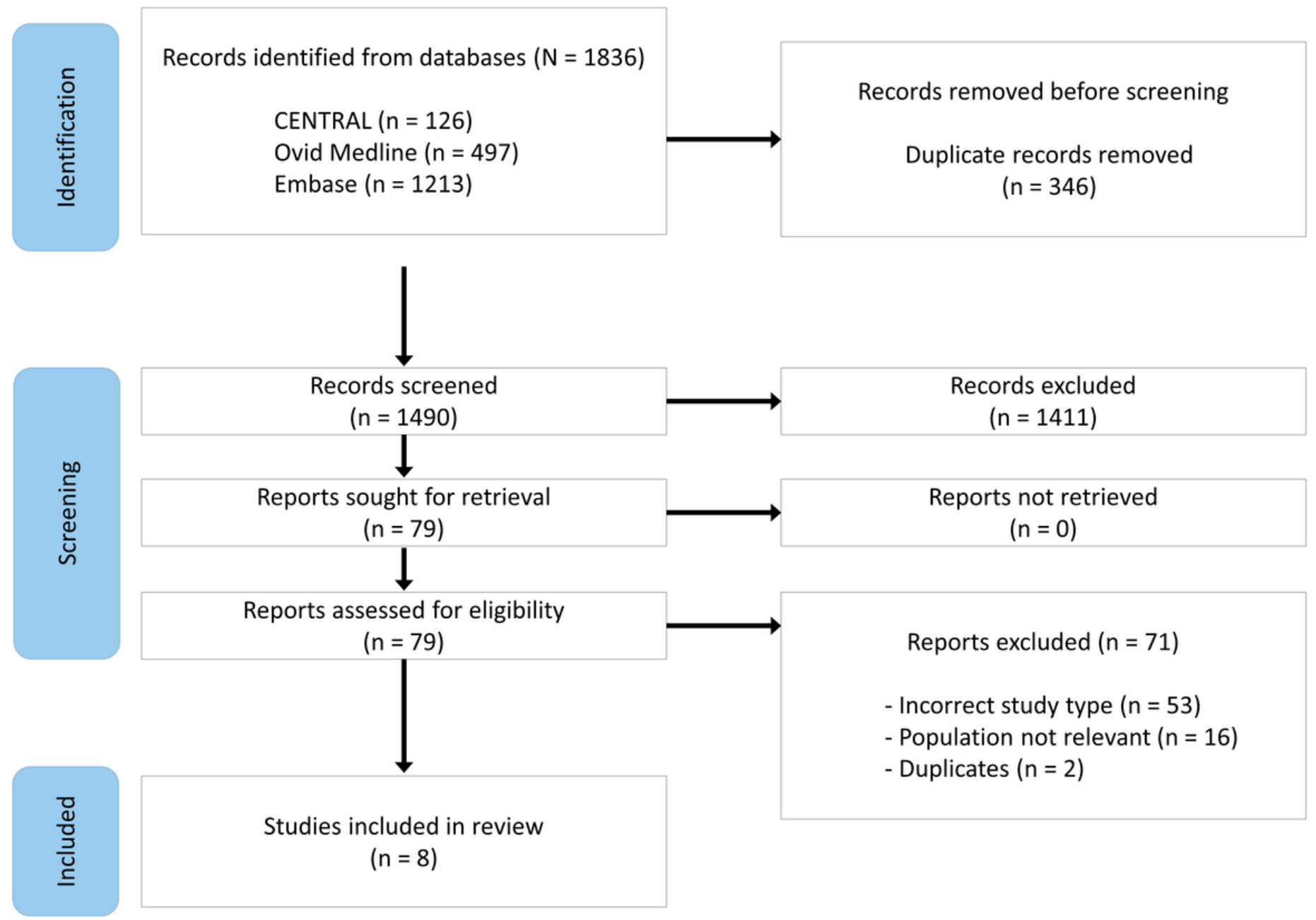

Figure 1

PRISMA flowchart of paper screening process. 


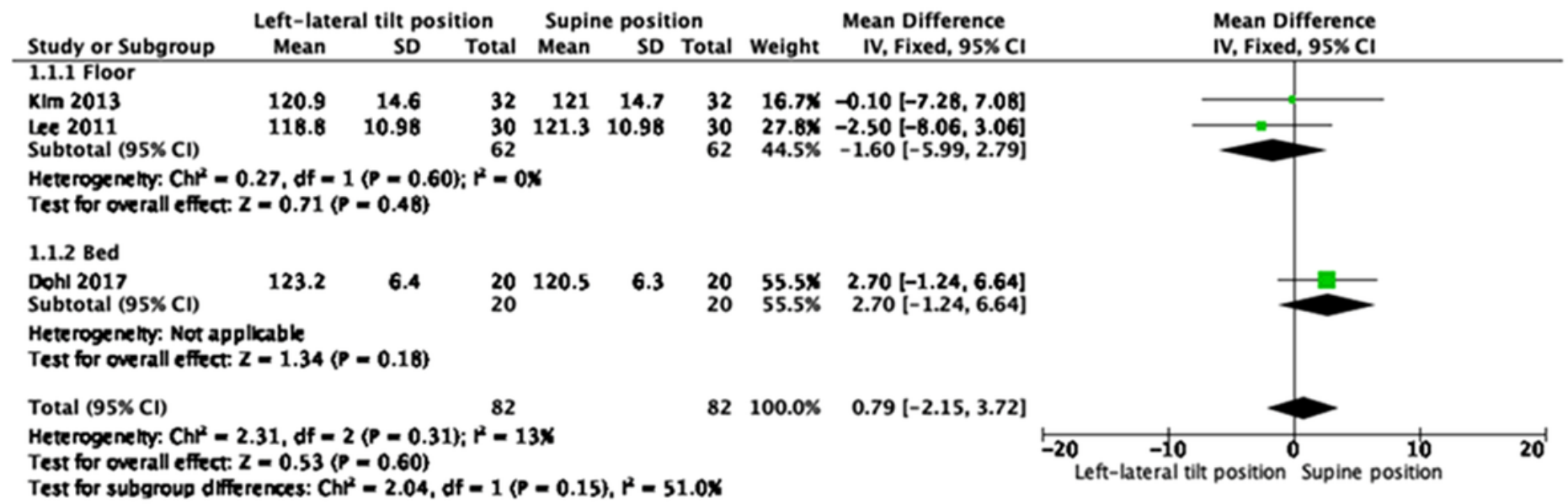

Figure 2

Meta-analysis of the mean difference in chest compressions per minute during resuscitation with the patient mannequin, showing no statistically significant difference between the left-lateral tilt position and the supine position. 


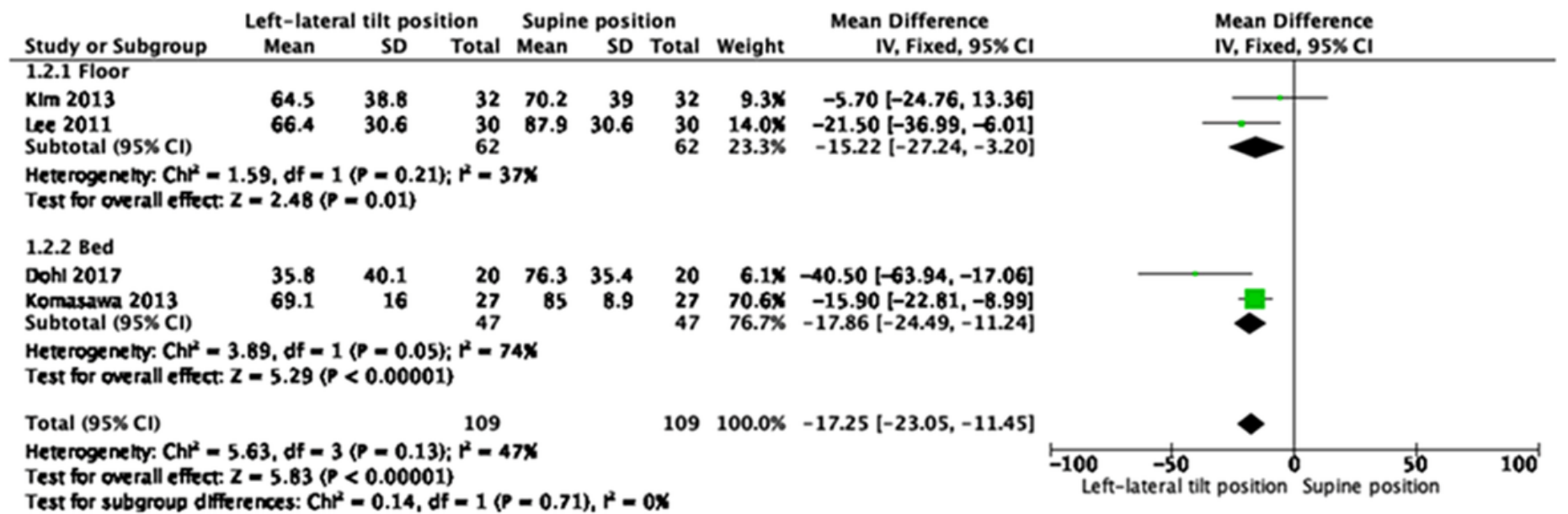

Figure 3

Meta-analysis of the mean difference in the rate of correct chest compression depth (\%) during resuscitation, showing a significantly lower rate (17.25\% lower) with the patient mannequin in the left-lateral tilt position than in the supine position. 


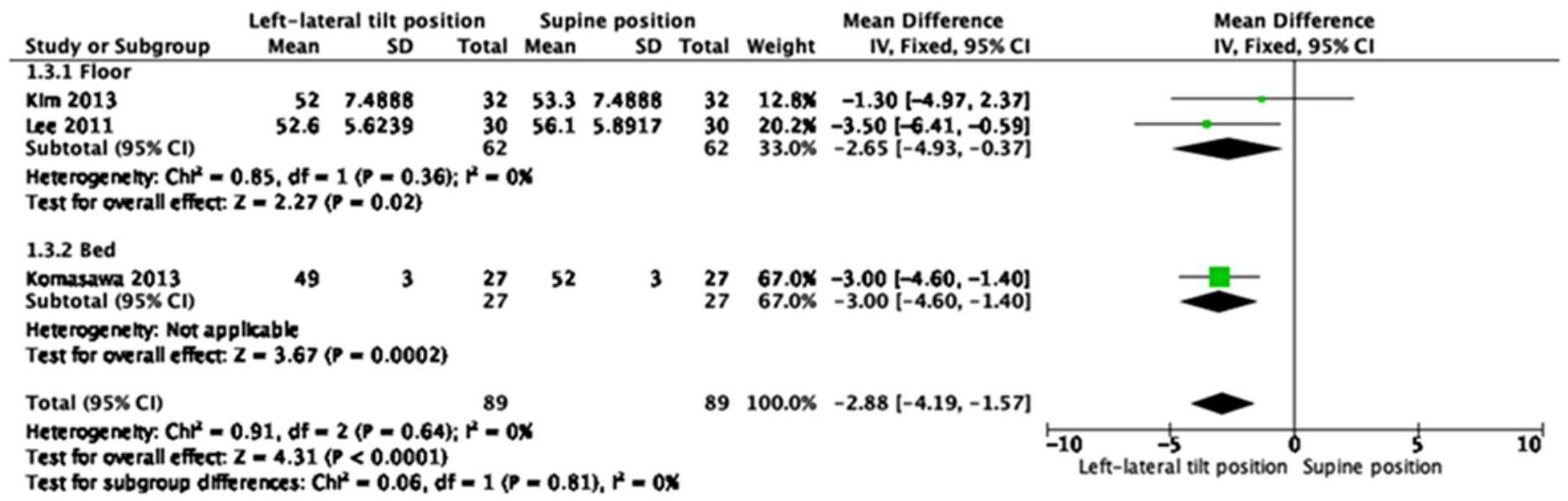

\section{Figure 4}

Meta-analysis of the mean difference in the chest compression depth ( $\mathrm{mm}$ ) during resuscitation, showing a significantly lower depth (2.88 $\mathrm{mm}$ lower) with the patient mannequin in the left-lateral tilt position than in the supine position. 


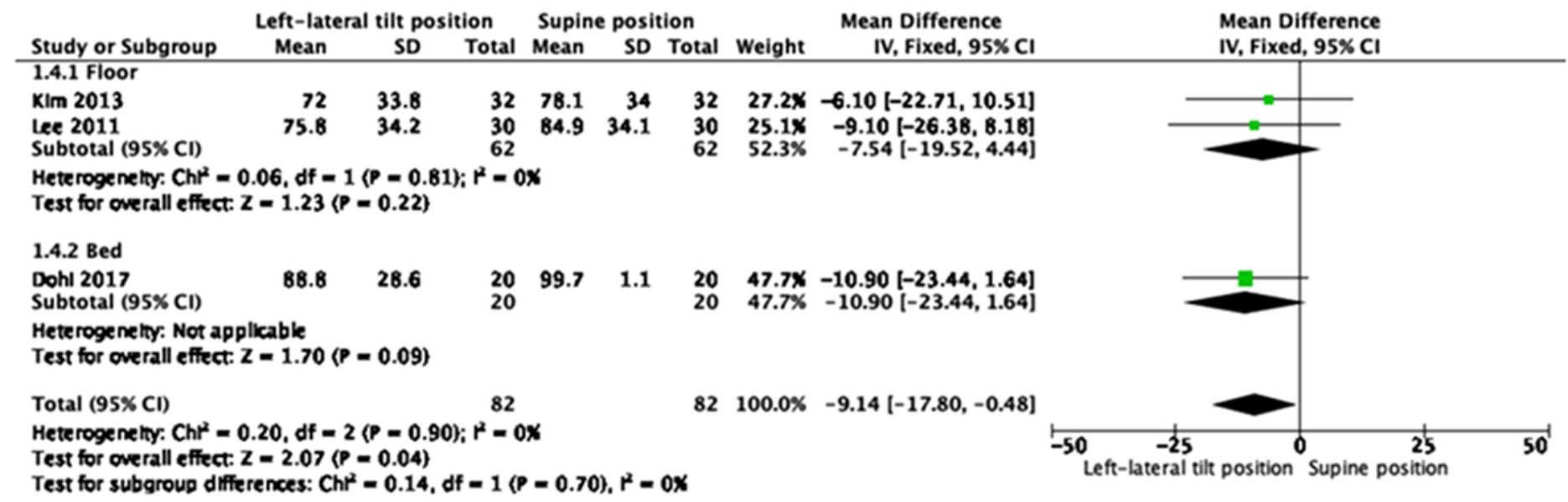

Figure 5

Meta-analysis of the mean difference in the correct hand position rate (\%) during resuscitation, showing a significantly lower rate (9.14\% lower) with the patient mannequin in the left-lateral tilt position than in the supine position.

\section{Supplementary Files}

This is a list of supplementary files associated with this preprint. Click to download.

- PRISMA2020checklist.docx 